SLAC -301

UC $-34 D$

(T)

\section{TWO EXERCISES IN SUPERSYMMETRY: A LOW-ENERGY SUPERGRAVITY MODEL AND FREE ऽTRING FIELD THEORY *}

\author{
Chriatian Richard Preitsehopf \\ Stanford Linear Accelerator Center \\ Stanford University \\ Stanford, Celifornia 94305
}

September 1986

Prepared for the Department of Energy under contract number DE-AC03-76SFO0515

Printed in the United States of America. Available from the National Technical Information Service, U.S. Department of Commerce, 5285 Port Royal Road, Springfeld, Virginia 22161. Price: Printed Copy A05, Microfiche A01.
TWO EXERCISES IN SUPERSYMMETRY:

A LOW-ENERGY SUPERGRAVITY MODEL

AND

FREE STRING FIELD THEORY

SLAC- -302

DE87 000346
Christian Richard Preitschopf, Ph.D.

Stanford University, 1986

This thesis consists of two parts. In the first we study the new features of a supersymmetric standard model in the presence of heavy families. We assume the minimal set of Higgs fields, the desert between the electroweak and the grand unification scale and perturbative values of the dimensionless parameters throughout this region. Using the numerical as well as the approximate analytic solution of the renormalization group equations, we study the evolution of all the pasameters of the theory in the case of large Yukawa couplings for the fourth family. The desired spontaneous symmetry breaking of the electroweak symmetry takes place only for a rather unnatural choice of the initial values of certain mass parameters at the grand unification scale. Two scenarios are possible, depending on the value of the gravitino mass. If it is smaller than $200 \mathrm{GeV}$ the vacuum expectation values of the Higgs fields emerge necessarily in an interplay of the tree level Higgs potential and its quantum corrections and are approximately equal. The quark masses of the fourtis family are roughly $135 \mathrm{GeV}$, while the mass of the fourth charged lepton has an upper bound of $90 \mathrm{GeV}$. Further characteristic features of this scenario are one light neutral Higgs field of mass $50 \mathrm{GeV}$ and gluino masses below $75 \mathrm{GeV}$. If the gravitino mass is higher than $200 \mathrm{GeV}$ one obtains a scaled up version of the well-known three family, heavy top scenario with quark masses between 40 and $205 \mathrm{GeV}$ and all superparticle masses heavier than $150 \mathrm{GeV}$ except the photino, gluino, one chargino and one neutralino.

* Ph.D. Theij. 
In the second part of the thesis we generalize the gauge-invariant theory of the free bosonic open string to treat closed strings and superstrings. All of these theories can be written as theories of string differential forms defined on suitable spaces. All of the bosonic theories have exactly the same structure; the Ramond theory takes an analogous first-order form. We show explicitly, using simple arid general manipulations, how to gauge-fix each action to the light-cone gauge and to the Feynmar-Siegel gauge.

\section{ACKNOWLEDGEMENTS}

The story of this thesis begins in Germany. Had it not been for the encouragement of Jorrit de Boer and the generous support by the Fũrst von Thurn und Taxis, the Stiftung Maximilianeum, the State of Bavaria and the Studienstiftung des Deutschen Volkes, I would not have crossed the Atlantic.

I am indebted to my fellow graduate students, from and with whom I learned a great deal, and who knew how to throw a decent party. Those who stand out in my memory are Bill Keirstead, Chris Wendt, Gregory Athanasiu, Joseph Atick, Kent Hornbostel, Marc Carter, Mike Mattis, Peter Arnold, Rene Ong and Richard Ward.

The Mahurins were my family while I worked on this thesis, and for that I am deoply grateful.

1 have profited from discusssions with Howard Haber, Jonathan Bagger, Marc Sher, Marek Karliner, Marvin Weinstein, Savas Dimopoulos and Ulrich Ellwanger. Thanks to all of them.

This thesis is the result of work done in collaboration with Mirjam Cretic (chapter II.) and Dan Friedan and Emil Martinec (chapter III.). I am grateful for thelr contributions.

Finally, I wish to thank Tom Banks and Michael Peskin. Both of them appeat on the list of authors of chapter III. Their influence, however, goes far beyond the many hours of discussions we spent in the course of that work. Tom taught me quantum field theory in an excellent course, and as my adviser Michael introduced me to the finer points of the subject. I will not .. his enthusiasm for physics and the beautiful ad hoc lectures he would give to answer his students' questions. 


\section{DISCLAIMER}

This repon was prepared as an account of work sponsored by an agency of the United States Government Neither the United States Government nor any agency thereof, nor any of their cmprom employees, makes any warranty, express or implied, or assumes any legal liability or responsibility for the accuracy, completeness, or usefulness of any information, apparatus, produch, or process disclosed, or represents that its use would not infringe privately owned rights. Reiermanufacturer, or otherwise does not necessarily constitute or imply its endorsement, recommendation, or fauring by the United States Government of any agency thereol. The views and opinions of authors expressed herein do not necessarily state or reflect those of the United States Government of any agency thereof.

\section{INTRODUCTION . . . . . . . . . . . .}

1. Supersymmetry ................... . . 2

2. Model Building . . . . . . . . . . . . . . . . 4

3. Superstrings . . . . . . . . . . . . . . 5

References .....................6 6

II. A LOW-ENERGY SUPERGRAVITY MODEL . . . . . . . 7

1. Preliminaries ...................8

2. The Model

3. Evolution of the Parameters .

3.1 Gauge parameters and an Approximation Scheme . . . . . . 15

3.2 Parameters of the Superpotential . . . . . . . . . 17

3.3 Soft Supersymmetry Breaking Mass Parameters . . . . . . . . 21

4. Spontaneous Symmetry Breaking . . . . . . . . . . . . . 29

5. Mass Spectrum . . . . . . . . . . . . . . 36

6. Sumnary ....................... 39

Appendix A. Renormalization Group Equations . . . . . . . . . 41

Appendix B. Approximate Analytic Solution . . . . . . . . 45

References ................... 48

III. FREE STRING FIELD THEORY . . . . . . . . . . . 50

1. Overview. . . . . . . . . . . . . . 51

2. The String Exterior Derivative and the BRST Charge . . . . . . 54

3. The Open Bosonic String ................ . 60

4. The Closed Bosonic String . . . . . . . . . . . . 70 
5. Neveu-Schwarz Strioga . . . . . . . . . . . . . 74

6. Ramond Strings . . . . . . . . . . . . . . 76

Reference

\section{LIST OF FIGURES}

1. Gauge couplings in the case of four families ... . . . . . 16

2. Quark Yukawa couplings ................. 20

3. Electron Yukawa coupling . . . . . . . . . . . . . 21

4. The Higgs mass parameter $\mu \ldots . . . . . . . .22$

5. The soft Higgs mass parameter $m_{H_{4}} \ldots \ldots . \ldots . \ldots$

6. The Higgs mass parameters $m_{H_{1}, H_{2}}^{2} \ldots \ldots . \ldots . \ldots . \ldots$

7. The parameters $m_{Q_{L}, D_{H}, U_{H}}^{2} \ldots \ldots . \ldots . \ldots . \ldots 25$

8. Trilinear soft squark couplings . . . . . . . . . . . . . 27

9. Trilinear soft selectron couplings . . . . . . . . . . . . 28

10. The parameters $m_{E_{L}, E_{n}}^{2} \ldots \ldots \ldots . . \ldots . . . . . .29$

11. The Higgs potential ................ 34

12. Mass spectra ... . . . . . . . . . . . 38

\section{TABLES}

1. Parameters specifying numcrically solved examples 


\section{Supersymmetry}

Since its discovery in the early seventies ${ }^{[3]}$ superaymmetry has risen to quite a prominent plase in particle physics. It provides a symmetry operation transforming fermions into bosons, which has turned out to be a rather useful concept: we may explain small bosonic masses by linking the bosons to chiral and hence massless fermions, or use this symmetry to cancel certain radiative corrections to our theory, enabling us for instance to fix mass ratios to all orders in perturbation theory. There is hope that eventually a supersymmetric theory will be able to explain the huge gap between the weak interaction scale, i.e. $100 \mathrm{GeV}$, and the energies at which one will no longer see the difference befween the strong and the weak force, roughly $10^{14}-10^{17} \mathrm{GeV}$. Of course, it may turn out that nature has populated the intermediate region of energy scales. In this case it may be that supersymmetry will find its experimental verification not in particle physics, but in other areas such as nuclear physics ${ }^{\text {[?] }}$ or in solid state physics, in systems which at their critical point can be described as superconformal field theories in 2 dimensions.'

But let us be optimistic and believe in the concept of grand unification. Then we notice that we have to deal with physics uncomfortably close to the Planck Bcale, $10^{19} \mathrm{GeV}$, where we expect gravity to become an important force on microscopic scales. Our distress arises from the realization that the theoretical tools we used so successfully to describe physics up to the weak scale fail fundamentally when applied to gravity. At low energies this does not bother us too much, since we may always argue that we have set up only an effective theory valid at low energies and that the ultravjnlet infinities that arise and are thrown out in the process of renormalization deserve their fate because they are just an artifact of an approximation scheme which breaks down at very high energies.

At grand unified scales, however, we have lost most of of room we need to sweep such difficulties under the rug. Also, if we treat gravity as an ordinary quantum field theory, we run into severe problems: instead of alleviating it ac- tually worsens the set of problems associated with renormalization.

First attempts to solve this puzzle used a Fermi-Bose symmetry of gravity supergravity. ${ }^{[4]}$ The old problems did not plague these theories as seriously as they had pure gravity, but they persisted. It was the realization that supersymmetric string theories could provide finite, consistent quantum theories of gravity ${ }^{(0)}$ that finally saved the day.

These theories now provide at least one class of theories that has a chance of encompassing the theory of the world. Mioreover, string theories are strikingly beautiful, and in the absence of any experimental evidence for their validity for some time to come, this has to be regarded as one of the main reasons for their attractiveness as a description of nature. At the moment, there is of couse an immense interest in bringing strings into contact with the observed world, and to give an example, efforts ase under way to calculate the electron mass in orbifold compactification models. (") A whole new industry has sprung up, called superstring inspired models, ${ }^{[1]}$ that seeks to bridge the gap between what is known about strings at the Planck scale ind the physics at the weak scale we hope to explain. However, no model has yet emerged that yields a satisfactory connection between Planck scale physics and the standard model of electroweak and strong interactions at $100 \mathrm{GeV}$. 


\section{Model Building}

In the absence of a sufficiently successful top-down scenario of descibing physics, we are urged to find our way from the bottom up. Actually these days it is from the $Z^{0}$ up.

This is the process of building models: Explore the consequences of plausible assumptions about the structure of the world at energies higher than the weak scale and predict the consequences for experimentally accessible physics. At first we will invariably generate a great number of candidates for the model that best represents nature; this number may then be cut down by new experiments. However, many hypotheses which seem plausible a priori may be discarded or highly constrained by combining existing data with theoretical analysis. Chapter II. provides an example of this. I analyze the consequences of the assumtion that nature organizes itself in a way predicted by a quite general class of supergravity models, such that at the veak scale we would see just a minimal supersymmetric extension of the standard model, but with four families instead of the observed three. Does the weak scale emerge without introducing new uncomfortably large diminsionless numbers? Are we at liberty to organize the model in a way that it loọks almost unified at very high energies?

Questions like these already place quite substantial restrictions on our model, and we expect the upcoming experiments SLC, LEP and SSC to either verify or (more probably) kill it.

Chapter IL. consists of work done in collaboration with Mirjam Cvetic and has appeared in print. ${ }^{(1)}$

\section{Superstringe}

In contrast to the models mentioned above, string theorien will not be defintely verified or falsified very soon. Also, the constraints imposed by demanding internal consistency - finiteness and freedom of anomalies, ${ }^{\left[{ }^{[0]}\right.}$ do not reduce the number of allowed models to just one or very few, as was hoped early on. This hope was based on the fact that initially only a very stnall number of Calabi-Yau manifalds, which parametrize the low energy limit of string theories, was known. However, the great interest in these manifolds led to the rapid discovery of a large number of them, and each of these manifolds may serve as an approximate compactification of the superstring.

But then, there are quite a few aspects of string theory that are not well understood, and annong them we find in particular the question of what mechanism selects the correct ground state of string. This is a question one ought to ask in an off-shell setting of string theory, and almost all aspects of this 'string field theory' are currently the object of research. The free theories are now known and are described in section III., although some problems remain in the Ramond sector of elosed superstrings. Various proposals for the interactions have been brought forth, and at the moment it is far from clear whether they are equivalent and in which approach actual calculations can be performed. Again, in the absence of experimental knowledge we have substituted aesthetic arguments and searched for a formulation of the free string theories which looks elegant and pretty. From a unified point of view wc tried to conatruct covariant actions for all the free string fields. I do not claim complete success here, but let the reader judge which parts of chapter III. satisfy these requirements.

This chapter is based on the work of ref. 9, done in collaboration with $T$. Banks, D. Friedan, E. Martinec and M. Peskin. 


\section{References}

1. Yu. A. Gol'fand, E.P. Likhtman, JETP Letters 3,323 (1971); J. Wess, B. Zumino, Nucl. Phys. B70, 39 (1974); for a more complete set of references oee, e.g. P. Fayet, S. Ferrara, Physics Reports 32,249 (1977).

2. F. Tachello, Nucl. Phys. A374, 635C (1982).

3. D. Friedan, Z. Qiu, S. Shenker, Phys. Rev. Lett. 53, 1575 (1984); Phys. Lett. 151B, 37 (1985).

4. D. Z. Freedman, P. van Nieuwenhuizen, S. Ferrara, Phys. Rev. D13, 3214 (1976); S. Deser, B. Zumino, Phys. Lett.62B, 335 (1976).

5. T. Yoneya, Prog. Theor. Phys.51, 1907 (1974); J. Scherk, J, H. Schwarz, Nucl. Phys. B81, 118 (1974); M. B. Green, J. H. Schwarz, Phys. Lett. 149B, 117 (1985); Phys. Lett. 1518, 21 (1985).

6. L. Dixon, J. A. Harvey, C. Vafa, E. Witten, Nucl. Phys. B261, 678 (1985); L. Dixon, J. Harvey, C. Vafa, E. Witten, Strings on orbifolds 2, Print86-0246(1986); Shahram Hamidi, Cumrun Vafa, Interactions on orbifolds, HUTP-86/AO41 (1986); Kei Ito, Quark, lepton mass matrices in the string theory on orbifolds, UT-484-TOKYO (1986); Lance Dixon, Daniel Friedan, Emil Martinec, Stephen Shenker, The conformal field theory of arbifolds, EFI-86-42-CHICAGO (1986).

7. examples are: Brian R. Greene, Kelley H. Kirklin, Paul J. Miron, Graham G. Ross, $A$ superstring inspired standard model, Print-86-1012 (OXFORD) (1986); Darwin Chang, Rabindra N. Mohtepatra, A superstring inspired low-energy eelctroweak model, MdDP-PP-86-166 (1986).

8. M. Cretic, C.R. Preitschopf, . Nucl. Phys. B2Y2 , 490 (1986)

9. T. Barks, D. Friedan, E. Martinec, M. Peskin, C.R. Preitschopf, Nucl. Phys. B774, 71 (1986). 


\section{Preliminaries}

Locally supersymmetric gauge theories ${ }^{(1)}$ provide an attractive way of linking, though not truly unifying gravity with other forces of nature. It is very intriguing that $N=1$ supergravity $(S G)$ in ten dimensions $(d=10)$ arises $^{121}$ as an effective field theory of superstrings, ${ }^{(b)}$ which naturally incorporate gravity. Dimensionally reduced $N=1 \mathrm{SG}$ models in $d=4$ are therefore promising candidates for the effective theory which crosses the desert between the physics at the grand uninication mass scale and the physics at presently accessible energies. Those theories are very attractive because they provide the most satisfactory mechanism for spontaneous breaking of local supersymmetry (SS). ${ }^{\text {[d }}$ Realistic scenarios have been based on specific grand unified groups, ${ }^{(3)}$ the lefthanded electroweak symmetry $S U(2)_{L} \times U(1)_{Y}{ }^{[0-0]}$ and the leit-right symmetric group $S U(2)_{L} \times S U(2)_{R} \times U(1)_{B-L} L^{\mid 10.111}$. In these models the electroweak symmetry breaking is induced by the solt SS breaking terms which arise from the spontaneous breakdown of local SS. Mass parameters in these terms are of the order of the gravitino mass $m_{3 / 2}$ which therefore sets the weak scale, i.e., $M_{W}=O\left(m_{3 / 2}\right) \cdot[4,8,8,7] *$

If the soft SS breaking parameters do not evolve substantially frorn their initial values at $\mu_{R}=O\left(M_{P l}\right)$, one cannot break $S U(2)_{L} \times U(1)_{Y}$ at the tree level of the Higgs potential unless one is willing to introduce a highly unattractive Higgs singlet chiral superfield. Here $\mu_{R}$ is the renormalization scale and $M_{P l}$ is the Planck mass where local SS is broken. However, it is reasonable that the renormalization of these parameters is substantial and that it is this renormalization which at $\mu_{R}=O\left(M_{W}\right)$ triggers the spontaneous symmetry breaking (SSB) of the electroweak symmetry. In the heavy top scenario ${ }^{[r . s .10 \mid}$ a large Yukawa coupling of the top quark is responsible for this SSB. The Coleman-Weinberg scenario ${ }^{10.01}$ uses the idea that the parameters at $M_{W}$ leave the vacuum expectation values (VEV's) of the Higgs fields undetermined at the tree level of the patential unless

\footnotetext{
* In Ref. $11 \mathrm{~m}_{3 / 2}$ sets the scale of the right-handed vector boson.
}

one also includes radiative corrections of the Coleman-Weinberg type ${ }^{[22]}$ in the effective potential. In this case one ends up with s. light Higgs particle in the mass spectrum.

Here we study the effects of additional heavy families on the low energy $N=1$ SG theory in $d=4$ with the gauge group:

$$
G \equiv S U(2)_{L} \times U(1)_{Y} \times S U(3)^{C}
$$

We assume the desert between $M_{W}$ and the grand unification mass scale $M_{G}$ as well as perturbative values of the dimensionless parameters throughout this region. In particular we investigate the evolution of all the parameters according to the renormalization group equations (RGE's) when in addition to the usual $e-$, $\mu$ and $\tau$-families one has a heavier fourth family, consisting of the $\tau^{\prime}, b^{\prime}$ and $t^{\prime}$, with larger Yukawa couplings. ${ }^{\prime}$ Based on the mass pattern of the first three families it is a plausible hypothesis that the fourth family is a few times heavier than the third family. Therefore we assume that at $M_{G}$ the Yukawa couplings $h_{V_{1} D}$ for the fourth family are at least a few, i.e., $\geq 3$, times larger than the Yukara coupling of the top quark. Our Higgs sector is the minimal one with two $S U(2)_{L}$ doublet fields $\boldsymbol{H}_{1,2}$. For an appropriate choice of parameters, either the heavy top' or the Coleman-Weinberg scenario is realized, and we find distinctive mass spectra for both cases. It turns out that the latter is phenomenologically more attractive, giving rise to superparticles whose mass is within reach of experiments under construction. We therefore concentrate on the Coleman-Weinberg scenario, always keeping in mind that there is an aliernative.

A supersymmetric model with additienal heavy families may arise from family unification models or from the $E_{B} \times E_{8}$ heterotic string theory. ${ }^{|13|}$ Therefore an enalysis of the influence of such additional families may have implications for the low energy phenomenology of such theories.

I In the case of more than four families we lose asymplotic freedom for the strong interactions and $g_{3}$ diverges below $M_{G}$. 
In the nori-supersymmetric theory based on the gauge group $G$ a careful study ${ }^{\text {ic| }}$ of the RGE's has shown that the Yukawa couplings of heavy families approach an infrared-stable fixed point determined by the gauge couplings. ${ }^{\mid 16,10]}$ As we shall see the supersymmetric case shows similar features (see viso Ref. 17). This in turn implies that the mass parameters of the theory evolve in a specific way, constraining the theory at the weak scale. Thus, the nature of the SSB pattern and the particle spectrum exhibit characteristic features which tightly constrain.

This chapter is organized as follows. In Sec. 2 we specify the model and the assumptions and fix the notation. We devote Sec. 3 to a study of the renormalization group evolution of the parameters presenting the numerical results and explaining them via the approximate analytic solution. In Sec. 4, the SSB pattern of the electroweak symmetry is investigated $;$ the low energy mass spectrum is presenteo in Sec. 5. A summary is given in Sec. 6. For the sake of completeness we write down the complete set of the RGE's for our model in Appendix $A$. The approximate analytic solution is presented in Appendix B.

\section{The Model}

In this section we shall describe in detail the model and the assumptions used in the aralysis.

\section{Desert Hypothesis}

We assume the group $G$ of Eq. (1.1) to be the gauge symmetry of the theory between the weak scale $M_{W}=100 \mathrm{GeV}$ and the grand unification scale $M_{C}=2$. $10^{10} \mathrm{GeV}$. This enables us to study the undisturbed evolution of parameters over a wide range of energies from $M_{G}$ down to $M_{W}$. This allows certain parameters to reach an infrared fixed point to a good accuracy as $\mu_{R} \rightarrow M_{W}$, independent of their initial values.

Local SS is broken at $M_{P I} \sim 10^{18} \mathrm{GeV}$, thus giving rise to the soft SS breaking mass parameters. We ignore the renormalization of these parameters between $M_{P l}$ and $M_{G}$. In that way the number of the initial values of the free parameters in the theory does not proliferate.

Perturbative Unification

We assume that all the dimensionless parameters have perturbative values between $M_{W}$ and $M_{G}$. We are then allowed to analyze the RGE's using only one-loop beta functions.

\section{Particle Content}

We work with chiral superfields which transform under $S U(2)_{L} \times U(1)_{Y} \times S U(3)^{C}$ as follows:

$$
\begin{aligned}
\left(E_{L}\right)_{J} & =\left(\underset{\sim}{2},-\frac{1}{2}, 1\right) ;\left(E_{R}\right)_{J}=\left(\underset{\sim}{1}, 1, \frac{1}{\sim}\right) \\
\left(Q_{L}\right)_{J} & =\left(\underset{\sim}{2}, \frac{1}{6}, \underset{\sim}{3}\right) ;\left(U_{R}\right)_{J}=\left(\underset{\sim}{1}-\frac{2}{3}, \overline{3}\right) \quad\left(D_{R}\right)_{J}=\left(\underset{\sim}{1}, \frac{1}{3}, \overline{3}\right) \\
H_{1} & =\left(\underset{\sim}{2}-\frac{1}{2}, \underset{\sim}{1}\right) \quad H_{2}=\left(\underset{\sim}{2}, \frac{1}{2} \underset{\sim}{1}\right) .
\end{aligned}
$$

Here $f=1,2,3,4$ denotes the family index. The fourth family therefore transforms in the same way as the first three families. The Higgs superfields (2.1c) 
are the minimal set, for the supersymmetric extension of the standard model.

Superpotential

The most general renormalizable superpotential consistent with the particle content (2.1) has the following form:

$$
g=E_{R} \Gamma_{E} E_{L}^{T} \epsilon H_{1}+U_{R} \Gamma_{U} Q_{L}^{T} \epsilon H_{2}+D_{R} \Gamma_{D} Q_{L}^{T} \epsilon H_{1}+\mu H_{1}^{T} \epsilon H_{2}
$$

Here $\epsilon=\left[\begin{array}{cc}0 & 1 \\ -1 & 0\end{array}\right]$ and $\vec{\Gamma}_{E, U, D}$ are Yukawa matrices. Family indices are suppressed.

We neglect flavor-charging effects and therefore set the off-diagonal elements of $\Gamma_{E, U, D}$ to zero. The Yukawa couplings of the fourth family are assumed to be much larger than those of the other families.

$$
\left(\Gamma_{E, U, D}\right)_{44} \equiv h_{E, U, D} \gg\left(\Gamma_{E, U, D}\right)_{i i} ; \quad i=1,2,3
$$

However, $\left(\Gamma_{E, U, D}\right)_{i i}, i=1,2,3$, are not neglected in the RGE's. Out of the many examples we have analyzed on the computer, we pick five with initial values $h_{U}^{0} \geq h_{D, E}^{0} \approx(3$ to 20$) \cdot\left(\Gamma_{U}^{0}\right)_{33} \simeq\left(0.5\right.$ to 3.0 ) at $\mu_{G}$ in order to display the typical behavior of the relevant solutions of the R.GE's in graphs.

\section{Soft Supersymmetry Breaking Terms}

In addition to the supersymmetric part of the Lagrangian we include the most general soft SS breaking terms as they arise from the spontaneous breakdown of SG. 'These terms are of the following form:

$$
\mathcal{L}_{S}=\mathcal{L}_{0}+\mathcal{L}_{S 1}+\mathcal{L}_{S 2}
$$

where

$$
\mathcal{L}_{Q}=-\sum_{a=1}^{3} m_{\lambda_{a}} \lambda_{a} \lambda_{a}
$$

$$
\mathcal{L}_{S 1}=-\left[E_{R}\left(m_{E} \Gamma_{E}\right) E_{L}^{T} \epsilon H_{1}+U_{R}\left(m_{U} \Gamma_{U}\right) Q_{L}^{T} \epsilon H_{2}+D_{R}\left(m_{D} \Gamma_{D}\right) Q_{L}^{T} \epsilon H_{1}\right.
$$

$$
\begin{aligned}
& \left.+m_{H_{3} \mu} \mu H_{1}^{T} \epsilon H_{2}\right] \\
& \mathcal{L}_{S 2}=-\left[E_{L^{\prime}}^{\dagger} m_{E_{L}}^{2} E_{L}+E_{R}^{\dagger} m_{E_{R}}^{2} E_{R}+Q_{L}^{\dagger} m_{Q_{L}}^{2} Q_{L}+D_{R}^{\dagger} m_{D_{R}}^{2} D_{R}\right. \\
& \left.+U_{R}^{\dagger} m_{U_{R}}^{2} U_{R}+H_{1}^{\dagger} m_{H_{3}}^{2} H_{1}+H_{2}^{\dagger} m_{H_{2}}^{2} H_{2}\right] \text {. }
\end{aligned}
$$

The fields here denote the scalar components of the appropriate superfields. The subscript $a=1,2,3$ refers to the gauge group $U(1)_{Y}, S U(2)_{L}$ and $S U(3)^{C}$, respectively and again we have suppressed the family indices. The mass matrices $\left(m_{E, U, D} \times \Gamma_{E, U, D}\right.$ ) and $m_{E_{L}, E_{n}, Q_{L}, U_{R}, D_{n}}^{2}$ are chosen to be flavor diagonal. Here $m_{H_{1}}^{2}, m_{H_{2}}^{2}$ and $m_{H_{3}}^{2}$ denote the three mass parameters of the Higgs fields $H_{1,2}$ -

In order to get as close as possible to the experimentally determined values for the gauge coupling constants as extracted from Ref. [18], we set

$$
\sqrt{\frac{5}{3}} g_{1}^{0}=g_{2}^{0}=g_{3}^{0}=g_{0}=0.96
$$

at $M_{G}=2 \cdot 10^{10} \mathrm{GeV}$. This value is determined to about $1 \%$ to $2 \%$ by integrating the RGE's for our particle content. We also assume that at $M_{G}$ the soft SS breaking mass parameters have the following symmetry:

$$
\begin{aligned}
& m_{\lambda_{1}}=m_{\lambda_{2}}=m_{\lambda_{3}}=m_{\lambda}^{0} \\
& m_{H_{9}}=m_{H_{9}}^{0} \\
& m_{E}=m_{U}=m_{D}=m_{0} \\
& m_{H_{1}}^{2}=m_{H_{2}}^{2}=m_{E_{L}}^{2}=m_{E_{n}}^{2}=m_{Q_{L}}^{2}=m_{U_{R}}^{2}=m_{D_{n}}^{2}=m_{3 / 2}^{2} .
\end{aligned}
$$

Here $m_{H,}^{0}$ and $m_{0}$ are naturally of the order of the gravitino mass $m_{3 / 2}$, while the gaugino mass $m_{\lambda}^{0}$ is a free parameter, which can be smaller than $m_{3 / 2}$. This pattern of soft SS breaking mass parameters emerges from the hidden sector mechanism, ${ }^{|0|}$ which spontancously breaks the local SS at $M_{P l}$; by assumption the pattern persists down to $M_{G}$. 
Examples for typical initial values of the parameters at $M_{G}=2.10^{18} \mathrm{GeV}$ which ensure the proper spontaneous symmetry breaking pattern $\left|\left\langle H_{1}^{0}\right\rangle\right| \approx$ $\left|\left\langle H_{2}^{\circ}\right\rangle\right| \approx 123 \mathrm{GeV}$.

\begin{tabular}{|c|c|c|c|c|c|c|c|c|}
\hline & $h_{U}^{0}$ & $h_{D}^{0}$ & $h_{E}^{0}$ & $\pi_{3 / 2}$ & $m_{0}$ & $m_{H_{3}}^{0}$ & $m_{\lambda}^{0}$ & $\mu_{0}$ \\
\hline$(\mathrm{a})$ & 5 & 3 & 1 & 100 & 30 & 70 & 5 & 668 \\
\hline$(\mathrm{b})$ & 5 & 3 & 1 & 100 & 30 & 70 & 50 & 908 \\
\hline$(\mathrm{c})$ & 2 & 1 & 0.5 & 100 & 20 & 40 & 5 & 232.6 \\
\hline (d) & 0.5 & 0.5 & 0.3 & 40 & 20 & 30 & 30 & 85 \\
\hline$(\mathrm{e})$ & 3 & 3 & 1 & 200 & 200 & 200 & 200 & 3050 \\
\hline
\end{tabular}

The results are obtained by using the numerical solutions. Here $h_{E, U, D}^{0}$ denote the Yukawa couplings for the fourth family, $\mu_{0}$ is the mass parameter of the superpotential (see Eq. (2.2)), $m_{\lambda}^{0}$ and $m_{3 / 2}$ are the gaugino mass, and the gravitino mass, respectively while $m_{H_{3}}^{0}$ and $m_{0}$ denote the soft supersymmetry breaking mass parameters defined in $\mathrm{Eq} .(2.7 \mathrm{~b})$ and $(2.7 \mathrm{c})$. All the masses are in $\mathrm{GeV}$.
The coupling constants and the masses of our model evolve from the unification scale $M_{G}$ to the weak scale $M_{W}$ according to the renormalization group equations (RGE's) given in Appendix A. Their solution for the gauge couplings and gaugino masses are examined in Sec. 3.1. Results for the parameters of the superpotential and for the soft SS breaking mass parameters are presented in Sec, 3.2 and $\mathbf{3 . 3}$, respectively.

\subsection{Gauge Parameters and an Approximation Scheme}

The solution of the RGE's for the gauge couplings and gaugino masses with initial conditions (2.6) and (2.7a) is of the following form:

$$
\begin{aligned}
& g_{1}^{2}=\frac{g_{1}^{02}}{1-2\left(\frac{10}{3} N_{f}+1\right) g_{1}^{02} t} \\
& g_{2}^{2}=\frac{g_{2}^{02}}{1-2\left(2 N_{f}-5\right) g_{2}^{02} t} \\
& g_{3}^{2}=\frac{g_{3}^{02}}{1-2\left(2 N_{f}-9\right) g_{3}^{02 t}}
\end{aligned}
$$

and

$$
m_{\lambda_{i}}=m_{\lambda}^{0} \frac{g_{i}^{2}}{g_{i}^{02}} \quad i=1,2,3
$$

Here $t$ is related to the renosmalization mass scale $\mu_{R}$ in the following way:

$$
t \equiv \frac{1}{16 \pi^{2}} \ln \frac{\mu_{R}}{M_{G}}
$$

and $N_{f}$ denotes the number $n$ f families. If $N_{f}>4$ we lose asymptotic freedom for the strong interactions and $g_{3}$ diverges below $M_{G}$. This fact allows us to restrict our study to $N_{f}=4$. 


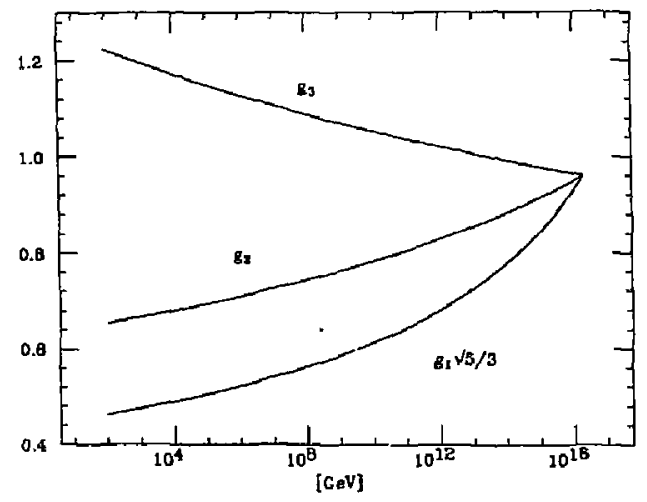

Fig.

Gauge couplings in the case of four families

We can now use the running, $g_{i}$ 's as parameters of other RGE's and numerically integrate those equations to find the evolution of other parameters, We have done that for a wide range of initial values and obtained accurate data for many cxamples, Unfortunaisly, the computer fails to give us adequate information regarding the generality of these results.

In our efforts to prove that we did not miss any phenomenologically attractive example in some corner of parameter space we found a way to obtain approximate analytic solutions for the RGE's (see $\Lambda$ ppendix $B$ ). They enable us to analytically relate parameter values at $M_{W}$ to those ai $M_{G}$ and thus to understand the computer results.

From Eqs. (3.1) one sees that $g_{2}^{2}$ and $g_{3}^{2}$ evolve slowly changing at most by a factor of two between $M_{G}$ and $M_{W}$. One may then expect to obtain a reasonably good approximate solution of the RGE's for other parameters if one sets for all
$\mu_{R}:$

$$
\begin{aligned}
g_{i} & \left.=\frac{1}{2} \mid g_{i}\left(\mu_{R}=M_{H^{H}}\right)+g_{i}^{0}\right] \quad i=1,2,3 \\
m_{\lambda_{i}} & =\frac{l}{2}\left[\frac{g_{i}^{2}\left(\mu_{R}=M_{W}\right)}{g_{i}^{02}}+1\right] m_{\lambda}^{0} i \quad i=1,2,3_{\mathrm{N}}
\end{aligned}
$$

where $g_{i}=0.55,0.81,1.09$ and $m_{\lambda_{i}}=0.62 m_{\lambda}^{0}, 0.73 \% \mu_{\lambda}^{0}, 1.31 m_{\lambda}^{0}$ for $i=1,2,3$, respectively. One also has $g_{1}^{2} \ll g_{2,3}^{2}$ and $g_{1}^{2}$ usually appears in the RGE's with a smaller coefficient than $g_{2,3}^{2}$. In most cases we ase allowed to neglect $g_{1}^{2}$ and $g_{1}^{2} m_{\lambda_{1}}^{2}$ compareu to $g_{2,3}^{2}$ and $g_{2,3}^{2} m_{\lambda_{2}, \lambda_{3}}^{2}$, respectively. In the approximate solution for the soft SS breaking mass parameters of the theory we performed an expansion in $\left(h_{U}-h_{D}\right) /\left(h_{U}+h_{D}\right)$ and neglected $h_{E}$ in comparison with $h_{U, D}$ i these two approximations are justified by the evolution of $h_{E, U, D}$ as given in Sec. 3.2. This will allow us to obtain to an accuracy of less than $10 \%$ the approximate analytic solution for the mass parameters $\mu$ (see Eq. (2.2)), $m_{H_{1}, H_{2}}^{2}$ and $\left(m_{Q_{L}, U_{R}, D_{R}}^{2}\right)_{44}$, which are important in determining the nature of the SSB pattern. For the sake of completeness the approximate anal; ، colution for other relevant mass parameters are stated in Appendix B. However, our approximations are generally good only to an accuracy of $10 \%$ to $30 \%$. In all cases we use the analytic estimates only to explain and substantiate the numerical results.

\subsection{Parameters of the Superpotential}

The evolution of the Yukawa couplings for the fourth family $h_{E, U, D}$ is gov, erned by the RGE's (A.5), (A.6) and (A.7), respectively. The numerical resulto for typical initial values of $h_{E, U_{,} D}^{0}$ at $M_{C}$ are presented for $h_{U, D}$ and $h_{E}$ in Fig. 2 and Fig. 3 , respectively. From those plots one secs that $h y$ and $h_{D}$ approach the same values $\sim 1.1$ at $M_{W}$ to an accuracy of $10 \%$, while the value of $h_{E}$ decreases as $\mu_{R} \rightarrow M_{W}$. This behaviour is quite independent of $h_{U, D}^{0}$, as shown by Bagger, Dimopoulos and Masso' ${ }^{\prime \prime \prime}$. In particular, we find that as long as $h_{l}^{0}, h_{D}^{0} \geq 0.5$, one obtains the same fixed point for any ratio of the two. In order to explain 
these observations we obtained the following approximate analytic expression for $h U, D, E$ :

$$
\begin{aligned}
& h_{U}^{2}=\frac{\tilde{g}^{2}}{1-X}\left[1+2 Z_{0}\left[\frac{X}{X_{0}}\left(\frac{1-X_{0}}{1-X}\right)\right]^{5 / 7}+O\left(Z_{0}^{2}\left[\frac{X}{X_{0}}\left(\frac{1-X_{0}}{1-X}\right)\right]\right)\right](3,6 a \\
& h_{D}^{2}=\frac{\bar{g}^{2}}{1-X}\left[1-2 Z_{0}\left[\frac{X}{\bar{X}_{0}}\left(\frac{1-X_{0}}{1-\bar{X}}\right)\right]^{5 / 7}+O\left(Z_{0}^{2}\left[\frac{X}{X_{0}}\left(\frac{1-X_{0}}{1-\bar{X}}\right)\right]\right)\right](3,6 b) \\
& h_{E}^{2}=h_{E}^{02}\left[\left(\frac{X}{X_{0}}\right)^{1-7}\left(\frac{1-X_{0}}{1-X}\right)\right]^{3 / 7} J(x)\left[1+O\left(Z_{0}^{2}\left[\frac{X}{X_{0}}\left(\frac{1-X_{0}}{1-X}\right)\right]\right)\right]
\end{aligned}
$$

where

$$
\begin{gathered}
X=X_{0} \exp \left(14 \bar{g}^{2} t\right), X_{0}=1-\frac{\bar{g}^{2}}{h_{0}^{2}} \\
h_{0}=\frac{h_{U}^{0}+h_{D}^{0}}{2}, \quad Z_{0}=\frac{h_{U}^{0}-h_{D}^{0}}{h_{U}^{0}+h_{D}^{0}} \\
\bar{g}^{2}=\frac{1}{7}\left(\frac{16}{3} g_{3}^{2}+3 g_{2}^{2}\right) \quad \eta=\frac{\theta_{2}^{2}+g_{1}^{2}}{\bar{g}^{2}} \\
J(x)=\left\{1+\frac{4}{7} \frac{h_{E}^{02}}{\bar{g}^{2}} \int_{X}^{X_{0}} \frac{d X}{X}\left[\left(\frac{X}{X_{0}}\right)^{1-\eta}\left(\frac{1-X_{0}}{1-X}\right)\right]^{3 / 7}\right\}^{-1}
\end{gathered}
$$

and $t$ is defined in Eq. (3.3). Here $O$ refers to the initial values of parameters at $M_{G}$ and $g_{1,2,3}$ denote the average values for the gauge couplings defined by Eq. (3.4). Equations $(3.6 \mathrm{a}, \mathrm{b})$ show that $h_{U}$ and $h_{D}$ approach the same infrared fixed point:"

$$
\lim _{\mu h \rightarrow 0} h_{U, D}=\bar{g}=1.09 .
$$

One sees that at $\mu_{R}=100 \mathrm{GeV}, X / X_{0}$ is a very small quantity, i.e., $X / X_{0}=$ $0.031<1$. Therefore, even for $Z_{0} \simeq 1$ the correction terms in Equs. (3.6) are smail and our approximation should make sense. We then find just as in our numerical solutions that as long as $h_{U}^{0}, h_{D}^{0} \geq 0.5$, the Yukawa couplings $h_{U, D}$ assume their asymptotic values (3.8) at $M_{W}$ to an accuracy of $10 \%$. From Egs. $(3.7 a, b)$ as well as from the numerical solution one also sees that this resilt persists even in the case $h_{U}^{0}>h_{D}^{0}$, as long as $h_{D} \geq 0.5$. For comparison, $\left(T_{U}^{0}\right)_{33}$ is of order 0.15 for the top quark with a mass around $40 \mathrm{GeV}$. This implies that $\left(\Gamma_{U}\right)_{33}$ contributes to RGE's only as a small correction to the leading contribution from $h_{U_{1} D}$, and the top mass becomes a free parameter of the model.

O7 the other hand from (3.6c) one sees that $h_{E}$ decreases as $\mu_{R} \rightarrow M_{W}$ and it has the infrared fixed point $h_{E}=0$. However, this value is not reached at $M_{W}$ because $\left[\left(X / X_{0}\right)^{1-\eta}\right]^{3 / 7} \sim 0.75 \sim O(1)$. Instead we can obtain an estimate $h_{E}^{2}\left(\mu_{R}=M_{W}\right)=\operatorname{Min}\left(h_{E}^{02}, \bar{g}^{2}\right) \times O\left(\frac{1}{2}\right)$. In Figs. 2 and 3 we also plot the approximate formulae (3.6) (dashed line) for $h_{U, D}$ and $h_{E}$, respectively. One sees that these solutions are in good agrecment with the numerical results.

The evolution properties of $h_{E, U, D}$ also justify the approximation that in the analytic solution for the evolution of the soft $5 S$ breaking mass parameters we performed an expansion in $\left(h_{U}^{0}-h_{D}^{0}\right) /\left(h_{U}^{0}+h_{D}^{0}\right)$ and neglected $h_{E}$ in comparison with $h_{U, D}$.

The Yukawa couplings of the first three families are small compared to $\bar{g}=$ 1.09 , i.e., $\left(\Gamma_{E, U, D}\right)_{i i} \ll \bar{g}$ where $i=1,2,3$. They have the characteristic feature that wher. $\mu_{R} \rightarrow M_{W},\left(\Gamma_{E}\right)_{i i}$ decrease, while $\left(\Gamma_{U, D}\right)_{i i}$ increase.

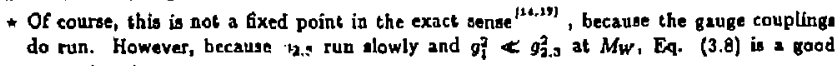
approximation. 


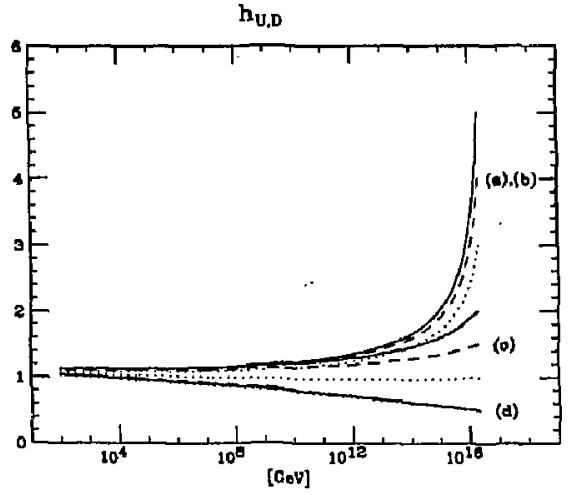

Fie.2

Exact solution for the evolution of $h_{U}$ (solid line) and $h_{D}$ (dotted line), the Yukawa couplings for the upand down-quarks of the fourth family. The dashed line denotes the approximate analytic solution for $h=$ $\left(h_{y}+h_{D}\right) / 2$. The initial values for $h_{U_{D}}$ at $M_{G}$ are taken from examples (a-d) of Table I.

From Eq. (A.8) we see that the mass parameter $\mu$ decreases and approaches the infrared fixed point $\mu=0$. However, at $\mu_{R}=M_{W}, \mu$ need not reach the fixed point, especially when $\mu_{0}$ is large compared to the other mass parameters and $h_{U, b}^{0} \dot{b}$ are not much larger than $O(\bar{g})$. This argument is supported by analyzing the form of the analytic estimate (B.4), which is in good agreernent with the ful! numerical solution as seen in Fig. 4. In Sec. 4 we show how the passibility that $\mu$ does not reach the infrared fixed point at $100 \mathrm{GeV}$ allows us to break $S U(2)_{L} \times U(1)_{Y}$ down to $U(1)_{\mathrm{em}}$ at $\mu_{R}=100 \mathrm{GeV}$ without contradicting known phenomenology.

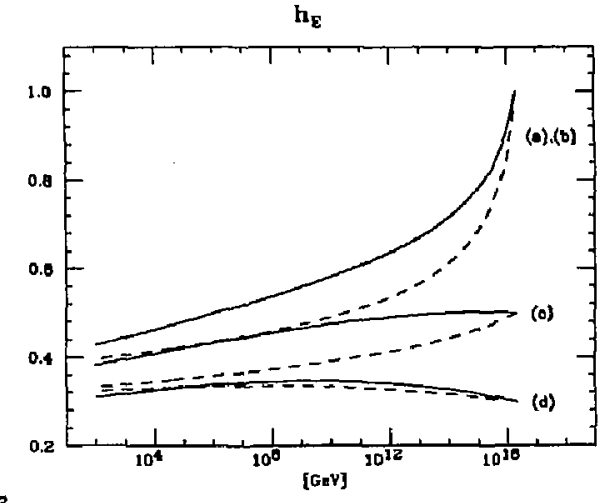

Fis.2

Evolution of $h_{E}$, the Yukawa coupling for the lepton of the fourth family. The numerical and anproximate analytic solution are plotted with the solid and dashed line, respectively. The values for the Yukawa couplings at $M_{G}=2 \cdot 10^{16} \mathrm{GeV}$ are chosen from examples (a-d) of Table I.

\subsection{Soft Supersymmetry Breaking Mass Parametere}

The RGE's for the soft SS breaking mass parameters are complicated (see Appendix A). We are especially interested in the infrared behavior of those parameters which are relevant for the proper breaking of $S U(2)_{L} \times U(1)_{Y}$ down to $U(1)_{\mathrm{cm}}$. These are the mass parameters $m_{H_{2}}, m_{H_{1}}^{2}$ and $m_{H_{2}}^{2}$ which appear in the terms with the doublet fields $H_{1,2}$, only (see Eqs. $(2.3 \mathrm{~b}, c)$ ). However, we shall also comment on the evolution of other SS breaking parameters which are relevant for the particle mass spectrum of sleptons and squarks.

From Eq. (A.12) one sees that the value of $m_{H_{3}}$ decreases as $\mu_{R} \rightarrow M_{W}$ and 
$\mu$

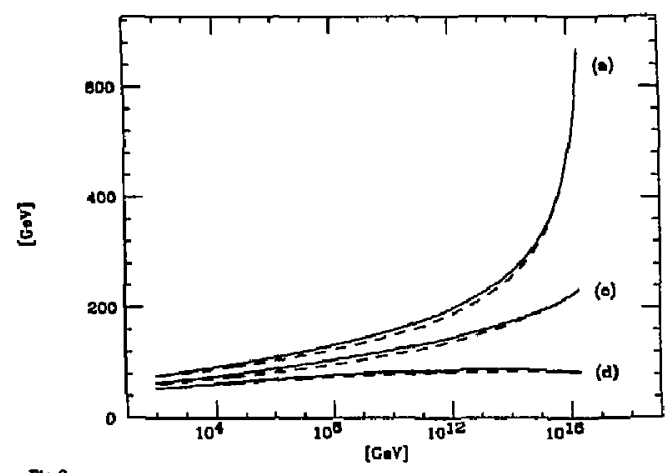

Tes

Numerical solution (solid line) and approximate analytic solution (dashed line) for $\mu$, the mass parameter of the superpotential (see Eq. (2.2)). The initial values for $h_{E, U, D}$ and $\mu$ at $M_{G}$ are chosen from the set (a), (c) and (d) of Table I.

jts value at $M_{w}$ depends linearly on its initial value $m_{H,}^{0}$ at $M_{G}$. This behavior can also be seen from the analytic solution (B.5a). Since $m_{H_{3}}^{0}$ does not appear in the evolution equations for other mass parameters its value is not restricted; thus $\mathbf{m}_{H_{x}}$ remains a free parameter of the model.

The RGE's $(A .21,22)$ for $m_{H_{1}, H_{2}}^{2}$ together with the RGE's $(A .18,18,20)$ for $m_{Q_{L}, U_{n}, D_{n}}^{2}$-the squark masses for the fourth family reveal that neither $m_{H_{1}, H_{2}}^{2}$ nor $m_{Q_{L}, U_{n}, D_{n}}^{2}$ approach an infrared fixed point for nonzero gaugino masses. The parameters $m_{H_{1}, H_{2}}^{2}$ decrease, while $m_{Q_{2}, U_{R}, D_{n}}^{2}$ increases as $\mu_{R} \rightarrow M_{W}$. The numerical evolution for $m_{H_{1}, H_{2}}^{2}$ and $m_{Q_{L}, U_{n}, D_{n}}^{2}$ is plotted in Fig. 6 and Fig. 7 , respectively. From Fig. 6 one sees that on the scale $m_{3 / 2}$ the mass parameters

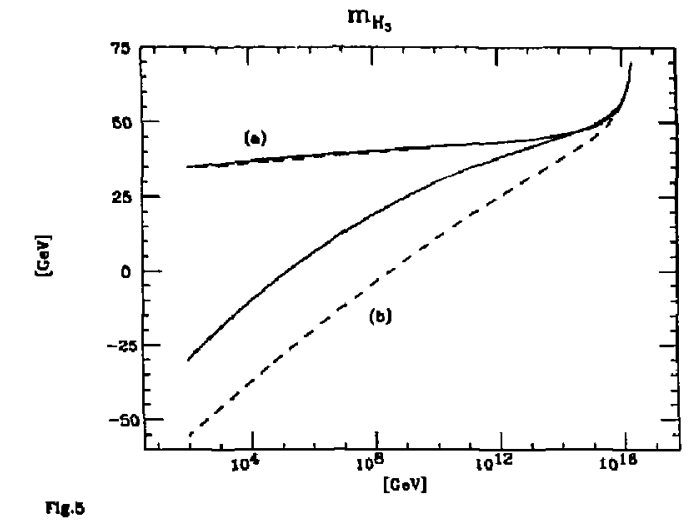

Numerical solution (solid line) and approximate analytic solution (dashed line) for $m_{H_{3}}$, (see Eq.(2.5b)). The initial values for the parameters at $M_{G}$ are frorn examples (a) and (b) of Table I.

$m_{H_{1}}^{2}$ and $m_{H_{3}}^{2}$ approach the same value as $\mu_{R} \rightarrow M_{W}$ even if at $M_{G}$ one has $h_{U}^{0} \neq h_{D}^{0}$ and $h_{E}^{0} \sim O(\bar{g})$. This is a consequence of the fact that as $\mu_{R} \rightarrow M_{W}$, $h_{U}$ and $h_{D}$ assume the same fixed point valuc and $h_{E}$ decreases. Also, in the RGE's $h_{E}^{2}$ appears with a smaller coefficient than the one in front of $h_{U, D}^{2}$. The latter arises from the color degrees of freedom. Therefore if one takes $h_{U} \approx h_{D}$ and $h_{E} \approx 0$ the RGE's for $m_{H_{1}}^{2}$ and $m_{H_{2}}^{2}$ become equivalent (see Appendix A) and then the evolution of these two parameters is the same.

At this point, two comments are in order. Firstly, a splitting of $m_{H_{3}}^{2}$ and $m_{H_{3}}^{2}$ does exist and we can enhance it by choosing $h_{U}^{0}$ and $h_{D}^{0}$ such that $h_{U}(t)$ and $h_{D}(t)$ are different as long as possible. In practice, this means setting $h_{U}^{0} \approx 3.0$ and $h_{D}^{0} \approx 0.5$. Secondly, even if $\left(m_{H_{1}}^{2}-m_{H_{2}}^{2}\right) /\left(m_{H_{3}}^{2}+m_{H_{3}}^{2}\right)$ is quite small, we 
may choose $m_{3 / 2}$ large enough so that $m_{H_{1}}^{2}-m_{H_{2}}^{2} \approx m_{W}^{2}$. Of course, this requires $m_{s / 2} \rightarrow m_{W}$, and on the computer we obtain $m_{3 / 2} \geq 200 \mathrm{GeV}$, depending on $h_{U}^{0}, h_{D}^{0}$

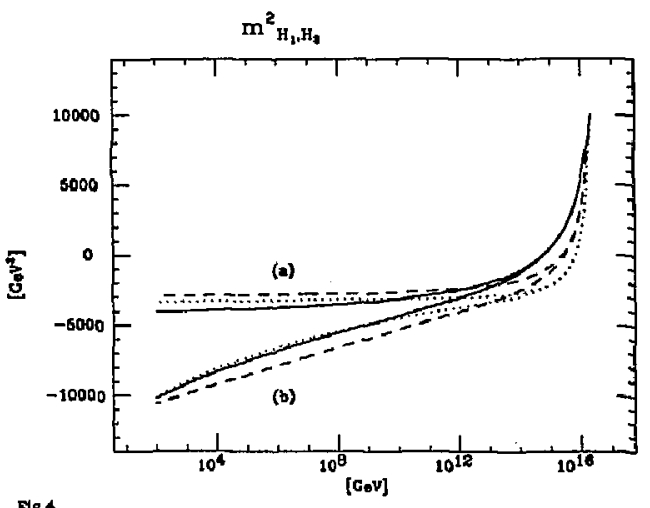

Fle.4

Numerical solution for the evolution of $m_{H_{1}}^{2}$ (solid line) and $m_{H_{3}}^{2}$ (dotted line) (see $\mathrm{Eq}$. (2.5c)) for the values at $M_{G}$ given in (a) and (b) of Table $I$. The dashed line denotes the approximate analytic solution for $m_{H_{+}}^{2}=$ $\left(m_{H_{1}}^{2}+m_{H_{2}}^{2}\right) / 2$.

$\cdot$

From Figs. 6,7 we also see that at $\mu_{R} \approx 100 \mathrm{GeV}, m_{H_{1}, H_{3}}^{2}$ are negative while $m_{Q_{L}, U_{R}, D_{R}}^{2}$ are strictly positive. This can be understood by examining the RGE's for $m_{H_{1}, H_{2}}^{2}$ and $m_{Q_{z}, U_{R,}}^{2} D_{R}$. Let us assume first that the gaugino masses are zero. In this case the relation between the beta functions for $m_{H_{1}, H_{2}}^{2}$ and $m_{Q_{2}, U_{R}, D_{R}}^{2}$ is

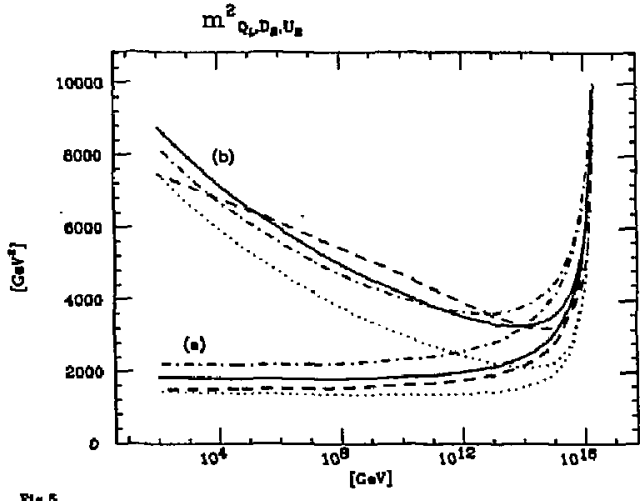

Numerical solution for the evolution of $m_{Q_{L}}^{2}$ (solid line), $m_{U_{n}}^{2}$ (dotted line) and $m_{D_{n}}^{2}$ (dot-dashed line), corresponding to the squark masses of the fourth family. The approximate analytic solution for $m_{S_{+}}^{2}=$ $\frac{1}{2}\left[m_{Q_{L}}^{2}+\frac{1}{2}\left(m_{U_{R}}^{2}+m_{D_{R}}^{2}\right)\right]$ is plotted with the dashed line. The initial values of the parameters are taken from examples (a) and (b) of Table I.

the following:

$$
\frac{d}{d t} m_{H_{1}, H_{2}}^{2} \approx \frac{3}{2} \frac{d}{d t} m_{Q_{h, U_{R}, D_{R}}^{2}} \geq 0
$$

Since $\frac{d}{d t} m_{H_{1}, H_{2}}^{2}>\frac{d}{d t} m_{Q_{L}, U_{R}, U_{D}}^{2}$ it follows that $m_{H_{1}, H_{2}}^{2}$ decrease at a larger rate than $m_{Q_{I}, U_{R}, D_{R}}^{2}$ and therefore $m_{H_{1}, H_{2}}^{2}<m_{Q_{L}, U_{R}, D_{R}}^{2}$ for all $\mu_{R}<M_{G}$. On the other hand we see from Eq. (B.6) that

$$
\frac{1}{2}\left(m_{H_{1}}^{2}+m_{H_{2}}^{2}\right)+m_{Q_{L}}^{2}+\frac{1}{2}\left(m_{U_{R}}^{2}+m_{D_{R}}^{2}\right) \approx m_{H_{1}, H_{2}}^{2}+2 m_{Q_{L}, U_{R}, D_{R}}^{2} \rightarrow 0
$$


as $\mu_{R} \rightarrow M_{W}$. This implies that at $M_{W}, m_{H_{3}, H_{2}}^{2} \approx-2 m_{Q_{L}, U_{R}, D_{R}}^{2}$ and therefore the Higgs masses $m_{H_{x} H_{7}}^{2}$ are necessarily negative while the squark masses are positive. This feature persists even in the case of nonzero gaugino masses because the beta function for $m_{Q_{L}, U_{A}, D_{n}}^{2}$ gets an additional negative contribution from gluino masses and it is therefore even smaller than the beta function for $m_{H, H}^{2}$.

The above aralysis is explained quantitatively by using the approximate analytic solutions (B.5b,c) for $m_{H_{1}, H_{1}}^{2}$ and $(B, 8 b, c)$ for $m_{Q_{2}, U_{R}, D_{n}}$ which are presented with the dashed line in Fig. 6 and Fig. 7, respectively. From the analytic estimates one can also obtain the following quantitative values for $m_{H_{1}, H_{2}}^{2}$ at $M_{W}$ as a function of initial values of mass parameters:

$$
\frac{1}{2}\left(m_{H_{1}}^{2}+m_{H_{2}}^{2}\right) \approx-0.28 m_{s / 2}^{2}-3.86 m_{\lambda}^{02}
$$

$$
\frac{1}{2}\left(m_{H_{1}}^{2}-m_{H_{2}}^{2}\right) \approx 0.078 \frac{Z_{0}}{h_{0}^{5 / 7}}\left(3 m_{3 / 2}^{2}-0.71 m_{0}^{2}+2.40 m_{\lambda}^{02}-4.25 m_{\lambda}^{0} m_{0}\right)
$$

Here $Z_{0}$ and $h_{0}$ are defined in Equs. (3.7b). The result that for any $h_{U}^{0}, h_{D}^{0} \geq 0.5$, at $\mu_{R}=100 \mathrm{GeV}$ the splitting is small on the scale of $m_{3 / 2}^{3}$, and the average mass square is negative and of order of the gravitino and/or gaugino masses has strong implications for the rature of SSB of $S U(2)_{L} \times U(1)_{Y}$. We discuss this in See. 4.

For the sake of completeness we also mention the evolution of the other soft Ss breaking parameters for the fourth family. The values for mu, increase as $\mu_{R} \rightarrow M_{W} W$ and they approach the same value $\sim 1.8 \mathrm{~m}_{\lambda}^{0}$. This behavior can also be seen from the analytic estimate (B.8a).

From Eq. (A.13) one sees that the value of $m_{E}$ decrezses as $\mu_{R} \rightarrow M_{W}$. If $h_{E}^{0}=O(\bar{g})$ and $m_{E}^{0}>m^{0} E_{k}, E_{n}$ it may be the case that at $\mu_{R} \sim 100 \mathrm{GeV}$ one ends up with $m_{E_{L_{1}} E_{R}}^{2}<0$ (see Eqs. (A.16,17)). In this case the solution which preserves $U(1)_{e m}$ is a saddle point, because the slepton masses are imaginary.

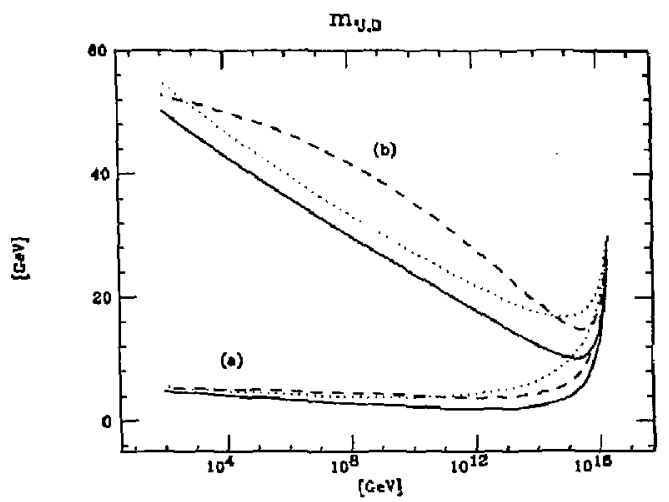

Fut. 8

The trilinear solt supersymmetry breaking parame ter mu (solid line) and $m_{D}$ (dotted line) corrspanding to the squarks of the fourth family (sce Eq. (2.5b)). The dashed line presents the approximate analytic solution for $m_{+}=\left(m_{U}+m_{D}\right) / 2$. The values of the parameters at $M_{G}$ are chosen from examples (a) asd (b) of Table J.

One may avoid such a pathological behavior by choosing the initial conditions $h_{E}^{0} \leqslant O(\bar{g})$ and/or $m_{E}^{0} \leqslant m_{E_{L}, E_{R}}^{0}$.

We briefly comment on the evolution of the soft SS breaking mass parameters for the first three families. The mass parameters $\left(m_{E, U, D} \times \Gamma_{E, U, D}\right)_{i,} i=$ $1,2,3$, are small compared to $m_{E, U, D} \times h_{E, U, D}$ because the Yukawa couplings $\left(\Gamma_{E, U, D}\right)_{i i}, i=1,2,3$, are snaller than $h_{E, U, D}$. The slepton and squark masses for the first three families $\left(m_{E_{L}, E_{R}, Q_{2}, U_{n}, D_{n}}^{2}\right)_{i i}, i=1,2,3$, evolve with a negative beta function which is in the leading order proportional to the product of the 


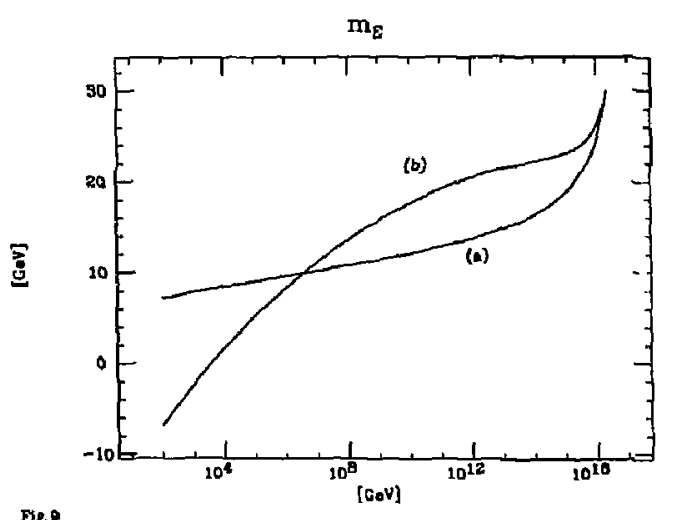

Fre.

Numerical solution for $m_{E}$, the trilinear soft supersymetry breaking mass parameter corresponding to the slepton of the fourth family (see Eq.(2.5b)). The inttial parametess at $M_{G}$ are from examples (a) and (b) of Table I.

squares of gaugino masses and gauge couplings. Therefore at $\mu_{R}=100 \mathrm{GeV}$ these masses are in general $m_{3 / 2}^{2}+O\left(m_{\lambda}^{02}\right)$.

For all the above mass parameters we obtained the numerical solution. Hown ever, since their numerical values are not essential for determining the nature of the SSB pattern, we do not present them.

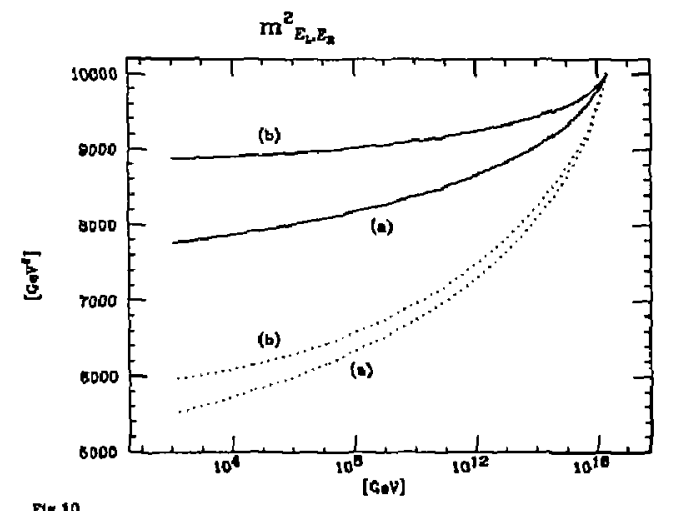

Tie 10

Numerical solution for $m_{E_{L}}^{2}$ (solid line) and $m_{E_{n}}^{2}$ (dotted line), the slepton masses of the fourth family (see Eq.(2.5c)). Parameters at $M_{G}$ are taken from examples (a) and (b) of Table I.

\section{Spontaneous Symmetry Breaking}

The fixed point behavior of large Yukawa couplings determines to a large extent the magnitude and the yrumetry pattern of the mass parameters in our model. In this section we study the implications for the spontaneous breakdown of $S U(2)_{L} \times U(1)_{Y}$ as they arise from the structure of the Higgs potential.

The SSB pattern should be compatible with the low-energy phenomenologs, therefore it should ensure $\left.M a x \mid\left\langle H_{1}^{0}\right\rangle,\left\langle H_{2}^{0}\right\rangle\right]=O\left(M_{W}\right)$ while the VEV'B of other scalar fields must be zero. Here the superscript 0 denotes the neutral component of the field. For the sake of further discussion we shall give here the part of the 
tree level potential which depends on the Higgs fields $H_{1,2}$ fields, only:

$$
\begin{aligned}
V_{T L}= & \left(m_{H_{1}}^{2}+\mu^{2}\right) H_{1}^{\dagger} H_{1}+\left(m_{H_{2}}^{2}+\mu^{2}\right) H_{2}^{\dagger} H_{2}-\mu m_{H_{3}}\left(H_{1}^{T} \varepsilon H_{2}+\text { h.c. }\right) \\
& \frac{g_{2}^{2}}{8} \sum_{a=1}^{3}\left(H_{1}^{\dagger} \tau_{a} H_{1}-H_{2}^{\dagger} \tau_{a} H_{2}\right)^{2}+\frac{g_{1}^{2}}{8}\left(H_{1}^{\dagger} H_{1}-H_{2}^{\dagger} H_{2}\right)^{2} .
\end{aligned}
$$

The mass parameters $\mu, m_{H_{8}}$ and $m_{H_{1}, H_{2}}^{2}$ are defined in Eqs. (2.2), (2.5b) and (2.5c), respectively, $\tau_{a}$ are Pauli matrices and $\epsilon=i \tau_{2}$

Let us first consider the heavy top' scenario of SSB. In Ref. [24] Hall shows that if $V_{T L}$ is bounded from below and its minimum breaks $S U(2) \times U(1)$, one obtains

$$
\left|m_{H_{1}}^{2}-m_{H_{2}}^{2}\right|=\frac{\left[\left(m_{H_{1}}^{2}+m_{H_{2}}^{2}+2 \mu^{2}\right)^{2}-\left(2 \mu m_{H_{3}}\right)^{2}\right]^{1 / 2}}{m_{H_{1}}^{2}+m_{H_{2}}^{2}+2 \mu^{2}}\left(m_{Z_{0}}^{2}+m_{H_{2}}^{2}+m_{H_{2}}^{2}+2 \mu^{2}\right)
$$

For sufficiently small $m_{H_{3}}$ we find $\left|m_{H_{1}}^{2}-m_{H_{3}}^{2}\right| \geq m_{Z_{0}}^{2}$ as a necessary condition for the heavy top' SSB pattern. A numerical study shows that its realization requires $m_{3 / 2} \geq 200 \mathrm{GeV}$ (see also $\mathrm{Eq}$.(3.11b)). In addition, to has to be chosen quite large ( $23 m_{3 / 2}$ ), while $m_{H_{3}}^{0}$ and $m_{0}$ must be kept small ( $\leq 50 \mathrm{GeV}$ ), otherwise we get transfered into a Coleman-Weinberd scenario with large $m_{3 / 2}$. We commment on the problems one then russ into at the end of section 5 . The larger $m_{3 / 2}$ is chosen the more freedom one hes with $m_{H_{b}}^{0}$ and $m_{0}$. The scenario necessitates introducing a rather large mass hierarchy that poses a formidable problem for model building. It leads to a distinctive mase opectrum, which we will discuss in section 5 .

\footnotetext{
- All the equarke and sleptans should have sero VEV'a. to the Higge potential those felde appear in the bilinear combination, and therefore the extremum equations are trivially atisfied. We ahall prove later that such a VEV pattern for squarke and sleptons abo iativfies contraints for the minimum.
}

Now, let us look at the case with $m_{3 / 2} \approx 100 \mathrm{GeV}$. Since then the RGE's lead to approximately equal values of $m_{H_{1}}^{2}$ and $m_{H_{2}}^{3}$ at $\mu_{R}=O\left(M_{W}\right)$ (see Eq. (3.11)), the minimization of $V_{T L}$ yields the VEV pattern:

$$
\left|\left\langle H_{1}^{0}\right\rangle\right|=\left|\left\langle H_{2}^{0}\right\rangle\right|=H / 2
$$

This pattern is correct up to order $\left(m_{H_{1}}^{2}-m_{H_{2}}^{2}\right) /\left(m_{H_{1}}^{2}+m_{H_{2}}^{2}\right) \leqslant 10 \%$ (see also Fig. 6).

The potential $V_{T L}$ as a function of the real VEV $H$ is then given by:

$$
V_{T L}=\frac{1}{2} m_{Y}^{2} H^{2}
$$

where

$$
m_{Y}^{2}=\frac{1}{2}\left(m_{H_{1}}^{2}+m_{H_{2}}^{2}\right)+\mu^{2}-\left|\mu m_{H_{3}}\right|
$$

Obviously, if $m_{Y}^{2}>0$ the system has a minimum at $H=0$, while for $m_{Y}^{2}<0$, $V_{T L}$ is unbounded from below. In such a situation one has to include quantum corrections to the tree level Higgs potential $V_{T L}$. This may be achieved by regarding $m_{y}^{2}$ as a function of $H$, i.e., $m_{W}^{2}=m_{H}^{2}\left(\mu_{R}=H\right)$, or by improving the potentiel à la Coleman-Weinberg. ${ }^{[12]}$ We thus observe that $m_{3 / 2}$ determines the nature of the SSB of $S U(2)_{L} \times U(1)_{Y}$ down to $U(1)_{e m ;}$ the SSB is necessarily radiative for $m_{3 / 2} \leq 200 \mathrm{GeV}$, i.c., quantum corrections to the tree level Higgs patential determine the magnitude of $H$

The stable minimum of the potential occurs at the scale $\mu_{R}$ where $m_{N}^{2} \sim 0$ and $H=O\left(\mu_{R}\right)$. From Eq. (3.11) one observes tha at $\mu_{R}=M_{W}, m_{H_{1}, H_{2}}^{2}$ are negative and large, i.e., of order of the gaugino and/or the gravitino mass. Also, $\mu$ approaches the fixed point value tero (see also Eq. (B.4)). T rerefore, $m_{X}^{2}$ is in general negative and large. This implies that the radiative $5 S B$ of $S U(2)_{L} \times U(1)_{r}$ takes place too early in the renarmalization group evolution, i.e, at $\mu_{R}>M_{W}$, yielding $H=O\left(\mu_{R}\right) \gg M_{W}$. This of course contradicts $H=245 \mathrm{MeV}$ which 
is obtzined from the experimentally obscrved $W^{ \pm}$and $Z^{0}$-boson masses. This implies that without eareful adjustments of the mass parameters at $M_{G}$, this ocenario is not comsistent with low energy phenomenology.

In order to obtain a realistic SSB pattern one has to choose $\mu_{0} \equiv \mu_{(}\left(\mu_{R}=M_{G}\right)$ in such a way that $m_{Y}^{2}\left(\mu_{R}=B\right)$ assumes a value close to zero, i.e., $m_{N}<$ $O\left(m_{\lambda}^{0}, m_{3 / 2}\right)$ at $M_{W}$. From the expression (4.4) for $m_{Y}^{2}$ and analytic estimates for $\mu, m_{H_{b}}$ and $m_{H_{1}, H_{2}}^{2}$ as given in (B.4), (B.5a) and (B.5b,c), respectively, one obtains the following constraint on $\mu_{0}$ in terms of the initial values $h_{0}=\left(h_{G}^{0}+\right.$ $\left.h_{D}^{0}\right) / 2, m_{\lambda}^{0}, m_{0}, m_{H_{2}}^{0}$ and $m_{3 / 2}$ (see Eqs. (2.7))

$$
\mu_{0} \approx 2.85\left(h_{0}\right)^{\theta / 2}\left(\sqrt{0.28 m_{3 / 2}^{2}+3.86 m_{\lambda}^{02}+0.25 m_{H_{3}}^{2}}+0.5 m_{H_{3}}\right)
$$

with $m_{H_{a}}$ being the value at $M_{W}$. In order to obtain the desired SSB pattern and to avoid the tuning of parameters one has to choose the following relations among the parameters at $M_{Q}$ :

$$
h_{U, D}^{0} \lesssim 5, \quad \mu_{0} Z 3 m_{3 / 2}, \quad m_{\lambda}^{0} \leqslant O\left(m_{3 / 2}\right)
$$

Also, $m_{H_{0}}^{0}$ has to be adjusted to ensure $m_{H_{3}} \ll m_{H_{3}}^{0}$ at the weak scale. If we relax any of the above constraints we have to introduce additional mass hierarchies in the model. Different typical initial values of the parameters at $M_{G}$ which yield $m_{N} \sim 0$ at $M_{W}$ are also obtained by using the numerical solution and are given in Table I. These values are in good agreement with the bounds (4.5) which were obtained from the analytic eatimates. To our knowledge there does not exist any model that satisfies naturally the constraint $\mu_{0} \gtrsim 3 \mathrm{~m}_{3 / 2}$. For example theories ${ }^{\mid \mathrm{al}}$ starting from a superpotential containing only dimensionless couplings cannot accommodate heavy families.

In the following we shall present the form of the quantum corrections to $V_{T L}$ and the minimization of the total potential. In the case when $H$ is larger than the soft $5 S$ breaking masses one can use the mass independent renormalization and sum all the powers of the leading logarithms. In this case one obtains the so-called renormalization group improved potential which is af the following form:

$$
V_{T}=\frac{1}{2} m_{M}^{2}\left(\mu_{R}=H\right) H^{2}
$$

In the leading logarithm approximation, $V_{T}$ has the following form:

$$
V_{T}^{1}=\frac{1}{2} m_{\lambda}^{2}\left(\mu_{R}=\mu_{L L}\right) H^{2}+V_{L L}
$$

where

$$
V_{L L}=\left.\frac{1}{2} \frac{d m_{Y}^{2}}{d t}\right|_{t_{L L}} \times H^{2} \frac{1}{16 \pi^{2}} \ln \frac{H}{\mu_{L L}}
$$

and $t=\frac{1}{16 \pi^{2}} \ln \frac{\mu_{L L}}{M 0}$.

At $\mu_{R}=O\left(M_{W}\right)$ one may actually encounter a situation when $H$ is of order of the largest soft SS breaking mass parameters. In this case the leading logarithm formula (4.7) is changed quantitatively and ass umes the following ColemanWeinberg form:

$$
V_{T}^{2}=\frac{1}{2} m_{\eta}^{2}\left(\mu_{R}=\mu_{L L}\right) H^{2}+V_{C W}
$$

where

$$
V_{C W}=\frac{3}{16 \pi^{2}}\left\{\sum_{i=1}^{2} \eta_{i}^{2} \ell n \frac{\eta_{i}}{\mu_{C W}^{2}}-\frac{1}{8} h^{4} H^{4} \ell n \frac{h^{2} H^{2}}{4 \mu_{C W}^{2}}\right\}
$$

and

$$
\begin{aligned}
\eta_{1,2} & =m_{S_{+}}^{2}+\frac{1}{4} h^{2} H^{2} \pm \sqrt{m_{S_{-}}^{4}+\frac{1}{4} h^{2} H^{2}\left(m_{+}-\mu \operatorname{sign} m_{H_{J}}\right)^{2}} \\
h & =\frac{h_{U}+h_{D}}{2} \\
m_{+} & =\frac{m_{U}+m_{D}}{2}
\end{aligned}
$$




$$
m_{S_{ \pm}}^{2}=\frac{1}{2}\left[m_{Q_{l}}^{2} \pm \frac{1}{2}\left(m_{D_{n}}^{2}+m_{U_{n}}^{2}\right)\right]
$$

The free parameter $\mu_{C W}$ is related to $\mu_{L L}$ in such a way that $V_{C W}$ is identical to $V_{L L}$ when $H$ is much larger than the soft SS breaking masses. In expression (4.10) we have included only the leading contribution to $V_{C W}$. All the parameters in $V_{L L}$ and $V_{C W}$ are taken at the renormalization scale $H_{L L}$.

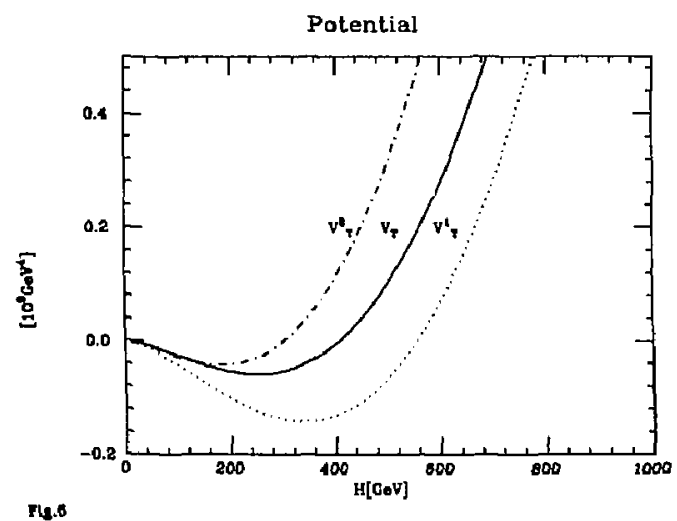

Fis.

Potentials $V_{T}, V_{T}^{1}$ and $V_{T}^{2}$ given by Eqs. (4.6), (4.7) and (4.9) as functions of $H=2\left|\left\langle H_{1}^{b}\right\rangle\right| \approx 2\left|\left\langle H_{2}^{0}\right\rangle\right|$. The sc-le $\mu_{L L}$ is chosen to be $7 \mathrm{TeV}$. For aesthetical reasons we subtract a constant from $V_{T}^{1}$ and $V_{T}^{2}$ so that $V_{T}$, $V_{T}^{1}$ and $V_{T}^{2}$ have the same value at $H=10 \mathrm{GeV}$. The initial values of parameters are taken from example (a) of Table 1 .

We choose $\mu_{L L}$ to be a few TeV. Then we may safely assume the mass in- dependent RGE's between $\mu_{L L}$ and $M_{G}$ while the leading logarithm potentials $V_{T}^{1}$ or $V_{T}^{2}$ still provide a good approximation at $M_{W}$. We compare $V_{T}, V_{T}^{1}$ and $V_{T}^{2}$ in Fig. 11. The location of the minimum is different in each case. However, this difference is not very significant, it can be countered by changing the initial value of the parameters at $M_{G}$ by a few percent. We may neglect the difference between $V_{T}, V_{T}^{1}$ and $V_{T}^{2}$ safely, since the two-joop corrections are expected to be of order $10 \%$. The minimization of $V_{T}$ yields a local minimum for the values of the parameters at $M_{G}$ given in Table I. We have checked numerically that this minimum is also the global minimum.

This version of our model (a heavy fourth family and the top quark having mass around $40 \mathrm{GeV}$ ) is very different frorn the models of Refs. $[7-9,19]$ with three families only and a heavy top quark mass $(Z 65 \mathrm{GeV})$. L. those models the large Yukawa coupling $\left(\Gamma_{v}\right)_{33}$ of the top quark determines $m_{H_{2}}^{2}$ to be negative at $M_{W}$ while $m_{H}^{2}$ is still positive at $M_{W}$. Therefore the electro-weak symmetry is broken already at the tree level of the Higgs potential with the pattern $\left\langle H_{2}\right\rangle \approx$ $245 \mathrm{GeV},\left\langle H_{1}\right\rangle \approx 0$. In our case the Yukawa couplings of the fourth family $h_{U, D} \geq 0.5$ are the dominant ones and they determine the pattern of $S \$ B$ while the contribution from $(\Gamma,)_{33}$ is only a small correction. 


\section{Mass Spectrum}

We compute the mass spectrum by diagonalizing numerically the tree level mass matrices with renormalization group improved parameters. Formulae for the masses are readily obtained form the Lagrangian described in Sec. 2 and have been given in the literature. ${ }^{|\neq 0|}$

\section{Heauy top' scenario}

In the heavy top' scenario our results coincide with what one would expect from a simple extension of three family models ${ }^{\text {(3.) }}$. For the fourth family quarks one obtains the BDM bound (Bagger-Dimopoulos-Masso ${ }^{11,117}$ ) $\tilde{m}_{U}, \tilde{m}_{D} \leq 205 \mathrm{GeV}$. Of course, previous bounds on the top mass no longer apply. It becomes an arbitrary parameter of the model. The only superparticles which can be naturally light are the gluino,photino and an additional neutralino which is mostly a $\tilde{h}^{0}$, as well as a chargino, a mixture of $\tilde{h}^{-}$and $\tilde{w}^{+}$. The lightest Higgs has a mass close to the electroweak gauge bosons. All the other superpartners have masses dictated by $m_{3 / 2} \approx 300 \mathrm{GeV}$ and are therefore safely out of range of detection by present experiments and those in construction.

\section{Coleman-Weinberg scenario}

If we assume the Coleman-Weinberg mechanism to be operative, the neutral Higgs nass matrix calculated from the tree level potential with renormalization group improved parameters has an imaginary eigenvalue. We replace it by the square root of the curvature of the potential $V_{T}$ (see Eqs. (4.6), (4.7) and (4.9)) at its minimum. Examples of mass spectra generated in that way are presented in Figs. 12.

Our model predicts the quark masses of the fourth family. In Sec. 3 we have seen that the Yukawa couplings $h_{U}$ and $h_{D}$ approach the same fixed point $\bar{g}=$ 1.09 at the weak scale. Since the only possible spontaneous symmetry breaking pattern is $\left|\left(H_{1}\right)\right| \approx\left|\left\langle H_{2}\right\rangle\right|=123 \mathrm{GeV}$, we obtain up- and down-quark masses of $135 \mathrm{GeV}$ to an accuracy of $10 \%$. From the approximate analytic solution (3.6c) for the evolution of $h_{E}$ we derive the approximate inequality $h_{E} \leq 0.65 \bar{g}$ and we get an uppes bound on the mass of the fourth lepton of $90 \mathrm{GeV}$. In most examples this mass is below $50 \mathrm{GeV}$.

Since $S U(2)_{L} \times U(1)_{Y}$ is broken radiatively, one neutral Higgs fields ends up light, i.e., in the range of $20-40 \mathrm{GeV}$.

In our model we are able to accommodate photino masses $m_{7}=0-40 \mathrm{GeV}$. As $m_{\bar{T}}$ rises the unpleasant mass hierarchy $\mu / m_{3 / 2}>3$ at $M_{G}$ tecomes even larger, as we easily see from Eq. (4.5). The model therefore prefers $m_{\bar{\gamma}} \leq 10 \mathrm{GeV}$. This in turn implies gaugino masses $m_{\lambda}, 575 \mathrm{GeV}$.

Barring any further fine-tuning of parameters all the other superparticles acquire masses of order $m_{3 / 2}$. The radiative symmetry breaking mecharism we employ decouples the value of $\left\langle H_{1}\right\rangle$ from that of $m_{3 / 2}$. This is illustrated by examples (a) and (e) of Table $I$ and Figs. 12a and 12b where we have chosen "' $m_{3 / 2}=100 \mathrm{GeV}$ and $m_{3 / 2}=200 \mathrm{GeV}$, respectively. In principle it is even possible to shift the masses of the superpartners of the ordinary particles into the $\mathrm{TeV}$ zegion. Apart from an increasingly difficult tuning of parameters we then have $\left\langle H_{1}\right\rangle / m_{3 / 2} \approx 0.1$ and two-loop effects must be taken into account. Also, since we may have $m_{H_{1}}^{2}-m_{H_{3}}^{2}=O\left(m_{W}^{2}\right)$, we are faced with the formidable problem of the Coleman-Weinberg analysis of a potential that is stabilized by quantum corrections in more than one direction.

It is also interesting to observe that for the case with $m_{3 / 2}=200 \mathrm{GeV}$ the mass of the lightest neutral Higgs field can be larger than the mass of $Z^{0}$ boson (see Fig. 12b). This differs from the resu]ts of Ref. [21] where the lightest neutral Higgs field cannot be heavier than $Z^{0}$ even in the case of radiative SSB. However, in our example the relation $\langle H\rangle\left\langle m_{3 / 2}\right.$ is different from the assumption of Ref. [21] where the soft supersymmetry breaking parameters are all of order or smaller than $M w$. 


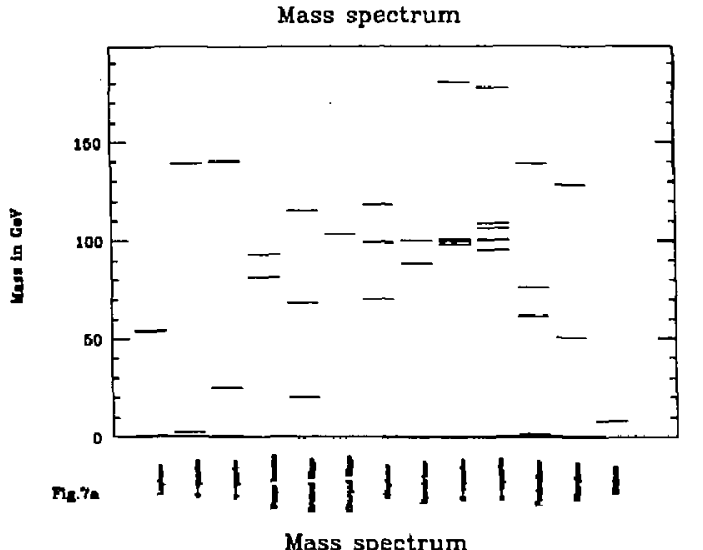

Mass spectrum

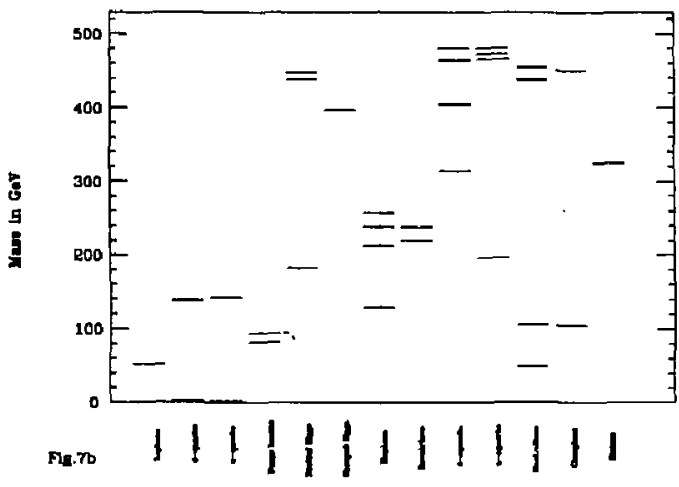

Particle mass spectrum of the model. The initial values of the parameters are chosen from examples (a) and (e) of Table I, for Fig. 12a and Fig. 12b, respectively.

\section{Summary}

We have studied a standard model in the context of $N=1$ supergravity when a heavy fourth family is present. The theory has a minimal set of Higgs fields with the two Higfs doublets. The fourth family is a replication of the first three families with the same gauge trassformation properties, but its Yukawa couplings $h_{U, D}^{0}$ at $M_{G}$ are chosen to be large, i.e., $\geq 0.5 .{ }^{*}$ We also assume that a desert extends between $M_{W}=100 \mathrm{GeV}$ and $M_{G}=2.10^{16} \mathrm{GeV}$ and that dimensionless parameters are perturbative through this region.

First we studied systematically the evolution of all the parameters of the theory from $M_{G}$ down to $M_{\mathrm{W}}$. We obtained the numerical solution which was illuminated by the approximate analytic solution. The Yukawa couplings and certain other mass parameters of the theory have an interesting infrared behavior: (i) the Yukaw couplings $h_{v}$ and $h_{D}$ for the up and down quarks of the four family approach the same infrared fixed point $\bar{g} \sim 1.1$, (ii) the masses $m_{H_{1}}^{2}$ and $m_{F_{2}}^{2}$ of the Higgs fields $H_{1}$ and $H_{2}$ decrease and approach a negative value which is of order of the gravitino or gaugino mass, with a splitting which is smaller by a factor of 10 and (iii) the mass parameter $\mu$ in the superpotential approaches the infrared fixed point 0. For $m_{3 / 2} \leq 200 \mathrm{GeV}$ the fact that $m_{H_{t}}^{2} \approx m_{H_{2}}^{2}<0$ at $\mu_{R}=M_{W}$ forces the sponteneous symmetry breakdown of the electroweak symmetry to occur in an interplay between the tree level Higgs potential and its quantum corrections. The spontaneous symmetry breaking pattern is then $\left\langle H_{1}\right\rangle \approx\left\langle H_{2}\right\rangle$. However, in order to ubtain $\left\langle H_{3}\right\rangle \approx 123 \mathrm{GeV}$ one has to choose unnatural initial values of the parameters at $M_{G}: \mu_{0} \gtrsim 3 m_{3 / 2}, h_{U, D}^{0} \leqslant 5, m_{\lambda}^{0} \lessgtr O\left(m_{3 / 2}\right)$ and $m_{H_{3}}^{0}$ has to be chrsen so that $\left|m_{H_{3}}\right| \ll m_{H_{3}}^{0}$ at $M_{W}$. For $m_{3 / 2} \geq 200 \mathrm{GeV}$ we get $\left\langle H_{1}\right\rangle \approx 0,\left\langle H_{21} \simeq 245 \mathrm{GeV}\right.$, provided $\mu_{0} \geq 3 m_{3 / 2}$ and $m_{H_{3}}^{0}, m_{0} \ll m_{3 / 2}$. It remains to be seen whether these constraints can be derived from a grand unifiec. theory.

*Te results persist for any $h_{u . D}^{0} \geq 0.5$, even in the case $h_{U}^{0}>h_{D}^{0}$ as long as $h_{D}^{0} \geq 0.5 \sim$ (2) to 3) $\left(\Gamma_{U}^{0}\right)_{39}$. Here $\left(\Gamma_{U}^{0}\right)_{33}$ is the top quark Yukawa coupling at $M_{G}$. 
Our model with heavy fourth family and the top quark mass around 40 $\mathrm{GeV}$ is different from the models of Refs. $(7-9,19)$ with three families only and a heavier top quark ( $\geq 65 \mathrm{GeV}$ ). In those models the SSB of the electro-weak symmetry takes place already at the tree level of the Higgs potential yielding the pattern $\left\langle H_{2}\right\rangle \simeq 245 \mathrm{GeV},\left\langle H_{1}\right\rangle \approx 0$.

Ous model also imposes interesting restrictions on the particle mass spectrum. In the heavy top' scenario, the fourth family quark masses have the BDM ${ }^{|14,17|}$ bound of $205 \mathrm{GeV}$. The lightest neutral Higgs has a mass around $m_{W}$. Only the gluino, photino, wiggsino and one other neutralino can be naturally light, while all the other superparticles acquire masses exceeding $150 \mathrm{GeV}$. In the ColemanWeinberg scenario the up and down quarks of the fourth farmily have the same mass $135 \mathrm{GeV}$ to an accuracy of $10 \%$, while the mass of the lepton has an upper bound of $90 \mathrm{GeV}$. Because of the radiative nature of the spontaneous symmetry breaking one ends up with one relatively light neutral Higgs field with a mass below $50 \mathrm{GeV}$. The gluino masses tend to be light, i.e., below $75 \mathrm{GeV}$. Masses of other particles, except fermions of the first three families, are in general in the region of $100 \mathrm{GeV}$.

We conclude that the large Yukawa couplings of the fourth family have strong implications on the low energy structure of the standard model within $N=1$ supergravity. They determine the spontaneous symmetry breaking pattern and restrict the particle mass spectrum.

\section{Appendix A. Renormalization Group Equations}

In the following we present the renormalization group equations for the model described in Sec. 2. They have been partially given in the literature. ${ }^{[27,3]}$ We have derived them by calculating the infinite parts of the one-loop diagrams in the superfield and component field formulation of the most general renormalizable softly broken super-Yang-Mills theory with chiral matter fields. The regularization method employed was dimensional reduction which is equivalent to dimensional regularization for our purposes. The difference between the two is proportional to $\epsilon=(4-d)$ and hence has no effect on the tesidues of the simple poles in c. Higher poles do not appear in a one-loop calculation.

Recently an independent evaluation using the effective potential approach has been given in Ref. [23], with identical results.

In the following equations we regard $\Gamma_{E, U, D},\left(m_{E, U, D} \times \Gamma_{E, U, D}\right)$ and $m_{E_{L}, E_{R}, Q_{L}, D_{R}, U_{R}}^{2}$ as matrices with family indices. $S$ is defined to be

$$
S=-m_{H_{1}}^{2}+m_{H_{2}}^{2}-t r m_{E_{L}}^{2}+t r m_{E_{n}}^{2}+t r m_{Q_{L}}^{2}+t r m_{D_{R}}^{2}-2 t r m_{U_{n}}^{2}
$$

and

$$
t=\frac{1}{16 \pi^{2}} \ln \frac{\mu_{R}}{M_{G}}
$$

while $N_{f}$ denotes the number of families.

Gauge couplings

$$
\begin{aligned}
& \frac{d}{d t} g_{1}=\left(\frac{10}{3} N_{f}+1\right) g_{1}^{3} \\
& \frac{d}{d t} g_{2}=\left(2 N_{f}-5\right) g_{3}^{3} \\
& \frac{d}{d t} g_{3}=\left(2 N_{f}-9\right) g_{3}^{3} .
\end{aligned}
$$




\section{Yukawa couplings}

$$
\begin{aligned}
\frac{d}{d t} \Gamma_{E}= & \Gamma_{E}\left(t r \Gamma_{E} \Gamma_{E}^{\dagger}+3 t r \Gamma_{D} \Gamma_{D}^{\dagger}-3 g_{1}^{2}-3 g_{2}^{2}\right)+3 \Gamma_{E} \Gamma_{E}^{\dagger} \Gamma_{E} \\
\frac{d}{d t} \Gamma_{D}= & \Gamma_{D}\left(t r \Gamma_{E} \Gamma_{E}^{\dagger}+3 t r \Gamma_{D} \Gamma_{D}^{\dagger}-\frac{7}{9} g_{1}^{2}-3 g_{2}^{2}-\frac{16}{3} g_{3}^{2}\right) \\
& +3 \Gamma_{D} \Gamma_{D}^{\dagger} \Gamma_{D}+\Gamma_{D} \Gamma_{U}^{\dagger} \Gamma_{U} \\
\frac{d}{d t} \Gamma_{U}= & \Gamma_{U}\left(3 t r \Gamma_{U} \Gamma_{U}^{\dagger}-\frac{13}{9} g_{1}^{2}-3 g_{2}^{2}-\frac{16}{3} g_{3}^{2}\right) \\
& +3 \Gamma_{U} \Gamma_{U}^{\dagger} \Gamma_{U}+\Gamma_{U} \Gamma_{D}^{\dagger} \Gamma_{D}
\end{aligned}
$$

The supersymmetric mass parameter $\mu$

$$
\frac{d}{d t} \mu=\mu\left(3 t r \Gamma_{U} \Gamma_{U}^{\dagger}+3 t r \Gamma_{D} \Gamma_{D}^{\dagger}+t r \Gamma_{E} \Gamma_{E}^{\dagger}-g_{1}^{2}-3 g_{2}^{3}\right)
$$

Gargino masses

$$
\begin{aligned}
& \frac{d}{d t} m_{\lambda_{1}}=2\left(\frac{10}{3} N_{f}+1\right) m_{\lambda_{1}} g_{1}^{2} \\
& \frac{d}{d t} m_{\lambda_{2}}=2\left(2 N_{f}-5\right) m_{\lambda_{2}} g_{2}^{2} \\
& \frac{d}{d t} m_{\lambda_{3}}=2\left(2 N_{f}-9\right) m_{\lambda_{3}} g_{3}^{2}
\end{aligned}
$$

The Mass parameter of the bilinear soft term

$\frac{d}{d t} m_{H_{3}}=2 t r \Gamma_{E}^{\dagger}\left(m_{E} \Gamma_{E}\right)+6 \operatorname{tr} \Gamma_{D}^{\dagger}\left(m_{D} \Gamma_{D}\right)+6 \operatorname{tr} \Gamma_{U}^{\dagger}\left(m_{U} \Gamma_{U}\right)-? m_{\lambda_{1}} g_{1}^{2}-6 m_{\lambda_{3}} g_{2}^{2}$

Mass parameters of the trilinear soft terms

$\frac{d}{d t}\left(m_{E} \Gamma_{E}\right)=4 \Gamma_{E} \Gamma_{E}^{\dagger}\left(m_{E} \Gamma_{E}\right)+5\left(m_{E} \Gamma_{E}\right) \Gamma_{E}{ }^{\dagger} \Gamma_{E}$
$+\left(m_{E} \Gamma_{E}\right)\left(\operatorname{tr} \Gamma_{E} \Gamma_{E}^{t}+3 \operatorname{tr} \Gamma_{D} \Gamma_{D}^{\dagger}-3 g_{1}^{2}-3 g_{2}^{2}\right)$

$+2 \Gamma_{E}\left[\operatorname{tr} \Gamma_{E}^{\dagger}\left(m_{E} \Gamma_{E}\right)+3 t r \Gamma_{D}^{\dagger}\left(m_{D} \Gamma_{D}\right)-3 m_{\lambda_{1}} g_{1}^{2}-3 m_{\lambda_{z}} g_{2}^{2}\right](A .13)$

$\frac{d}{d t}\left(m_{U} \Gamma_{U}\right)=4 \Gamma_{U} \Gamma_{U}^{\dagger}\left(m_{U} \Gamma_{U}\right)+2 \Gamma_{U} \Gamma_{D}^{\dagger}\left(m_{D} \Gamma_{D}\right)$

$+\mathbf{5}\left(m_{U} \Gamma_{U}\right) \Gamma_{U}^{\dagger} \Gamma_{U}+\left(m_{U} \Gamma_{U}\right) \Gamma_{D}^{\dagger} \Gamma_{D}$

$+\left(m U \Gamma_{U}\right)\left(3 t r \Gamma_{U} \Gamma_{U}^{\dagger}-\frac{13}{9} g_{1}^{2}-3 g_{2}^{2}-\frac{16}{3} g_{3}^{2}\right)+2 \Gamma_{U}\left[3 \operatorname{tr} \Gamma_{U}^{\dagger}\left(m_{U} \Gamma_{U}\right)\right.$

$\left.-\frac{13}{9} m_{\lambda_{1}} g_{1}^{2}-3 m_{\lambda_{2}} g_{2}^{2}-\frac{16}{3} m_{\lambda_{1}} g_{3}^{2}\right]$

$\frac{d}{d t}\left(m_{D} \Gamma_{D}\right)=4 \Gamma_{D} \Gamma_{D}^{\dagger}\left(m_{D} \Gamma_{D}\right)+2 \Gamma_{D} \Gamma_{U}^{t}\left(m_{U} \Gamma_{U}\right)$

$+5\left(m_{D}{ }_{D}\right) \Gamma_{D}^{\dagger} \Gamma_{D}+\left(m_{D} \Gamma_{D}\right) \Gamma_{U}^{\dagger} \Gamma_{v}$

$+\left(m_{D} \Gamma_{D}\right)\left(3 \operatorname{tr} \Gamma_{D} \Gamma_{D}^{\dagger}+\operatorname{tr} \Gamma_{E} \Gamma \varepsilon^{\dagger}-\frac{7}{9} g_{1}^{2}-3 g_{2}^{2}-\frac{16}{3} g_{3}^{2}\right)$

$2 \Gamma_{D}\left[t r \Gamma_{E}^{\dagger}\left(m_{E} \Gamma_{E}\right)+3 t r \Gamma_{D}^{\dagger}\left(m_{D} \Gamma_{D}\right)-\frac{7}{9} m_{\lambda_{1}} g_{1}^{2}\right.$

$\left.-3 m_{\lambda_{2}} g_{2}^{2}-\frac{16}{3} m_{\lambda_{3}} g_{3}^{2}\right]$

Soft mass squares

$\frac{d}{d t} m_{E_{L}}^{2}=\Gamma_{E}^{\dagger} \Gamma_{E} m_{E_{L}}^{2}+m_{E_{L}}^{2} \Gamma_{E}^{\dagger} \Gamma_{E}+2\left(m_{E} \Gamma_{E}\right)^{\dagger}\left(m_{E} \Gamma_{E}\right)+2 m_{H_{1}}^{2} \Gamma_{E}^{\dagger} \Gamma_{E}$

$2 \Gamma_{E}^{\dagger} m_{E_{n}}^{2} \Gamma_{E}+\left[-2 g_{1}^{2}\left|m_{\lambda_{1}}\right|^{2}-6 g_{2}^{2}\left|m_{\lambda_{2}}\right|^{2}-g_{1}^{2} S\right] 1$

$\frac{d}{d t} m_{E_{R}}^{2}=2 \Gamma_{E} \cdot \Gamma_{E}{ }^{T} m_{E_{R}}^{2}+2 m_{E_{n}}^{2} \Gamma_{E}{ }^{\prime} \Gamma_{E}^{T}+4\left(m_{E} \Gamma_{E}\right)^{*}\left(m_{E} \Gamma_{E}\right)^{T}$

$+4 m_{H_{1}}^{2} \Gamma_{E} \cdot \Gamma_{E}^{T}+4 \Gamma_{E}^{*} m_{E_{L}}^{2} \Gamma_{E}^{T}+\left.\left|-8 g_{1}^{2}\right| m_{\lambda_{1}}\right|^{2}+2 g_{1}^{2} S \mid 1$ 
$\frac{d}{d t} m_{Q_{L}}^{z}=\Gamma_{D}^{\dagger} \Gamma_{D} m_{Q_{L}}^{2}+m_{Q_{L}}^{2} \Gamma_{D}^{\dagger} \Gamma_{D}+\Gamma_{U}^{\dagger} \Gamma_{U} m_{Q_{L}}^{2}$

$+m_{Q_{L}}^{2} \Gamma_{U}^{\dagger} \Gamma_{U}+2 m_{H_{1}}^{2} \Gamma_{D}^{\dagger} \Gamma_{D}$

$+2 m_{H_{2}}^{2} \Gamma_{U}^{\dagger} \Gamma_{U}+2 \Gamma_{D}{ }^{\dagger} m_{D_{A}}^{2} \Gamma_{D}+2 \Gamma_{U}^{\dagger} m_{U_{A}}^{2} \Gamma_{U}$

$+2\left(m_{D} \Gamma_{D}\right)^{\dagger}\left(m_{D} \Gamma_{D}\right)+2\left(m_{U} \Gamma_{U}\right)^{\dagger}\left(m_{U} \Gamma_{U}\right)$

$+\left[-\frac{2}{9} g_{1}^{2}\left|m_{\lambda_{1}}\right|^{2}-6 g_{2}^{2}\left|m_{\lambda_{3}}\right|^{2}-\frac{32}{3} g_{3}^{2}\left|m_{\lambda_{1}}\right|^{2}+\frac{1}{3} g_{1}^{2} s\right]_{1}$

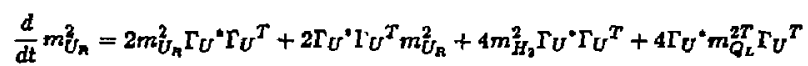

$+4\left(m_{V} \Gamma_{U}\right)^{*}\left(m_{U} \Gamma_{U}\right)^{T}+\left[-\frac{32}{9} g_{1}^{2}\left|m_{\lambda_{1}}\right|^{2}-\frac{32}{3} g_{3}^{2}\left|m_{\lambda_{3}}\right|^{2}-\frac{4}{3} g_{1}^{2} S\right]_{(A .19)}$

$\frac{d}{d t} m_{D_{R}}^{2}=2 m_{D_{n}}^{2} \Gamma_{D} \Gamma_{D} \Gamma^{T}+2 \Gamma_{D}{ }^{*} \Gamma_{D}{ }^{T} m_{D_{n}}^{2}+4 m_{H_{1}}^{2} \Gamma_{D}{ }^{*} \Gamma_{D}{ }^{T}+4 \Gamma_{D}{ }^{*} m_{Q_{L}}^{2 T} \Gamma_{D}^{T}$

$+4\left(m_{D} \Gamma_{D}\right)^{\circ}\left(m_{D} \Gamma_{D}\right)^{T}+\left[-\frac{8}{9} g_{1}^{2}\left|m_{\lambda_{1}}\right|^{2}-\frac{32}{9} g_{3}^{2}\left|m_{\lambda_{1}}\right|^{2}+\frac{2}{3} g_{1}^{2} s\right]_{(A .2 D)}$

$\frac{d}{d t} m_{H_{1}}^{2}=2 m_{H_{1}}^{2}\left(t r \Gamma_{E} \Gamma_{E}^{\dagger}+3 t r \Gamma_{D} \Gamma_{D}^{\dagger}\right)$

$+2 \operatorname{tr}\left(m_{E} \Gamma_{E}\right)\left(m_{E} \Gamma_{E}\right)^{\dagger}+6 \operatorname{tr}\left(m_{D} \Gamma_{D}\right)\left(m_{D} \Gamma_{D}\right)^{\dagger}$

$+2 \operatorname{tr} \Gamma_{E}\left(m_{E_{L}}^{2}+m_{E_{R}}^{2}\right) \Gamma_{E}^{\dagger}+6 \operatorname{tr} \Gamma_{D}\left(m_{Q_{L}}^{2}+m_{D_{R}}^{2}\right) \Gamma_{D}^{\dagger}$

$-2 g_{2}^{2}\left|m_{\lambda_{1}}\right|^{2}-6 g_{2}^{2}\left|m_{\lambda_{2}}\right|^{2}-g_{1}^{2} S$

$\frac{d}{d t} m_{H_{2}}^{2}=6 m_{H_{2}}^{2} t r \Gamma_{U} \Gamma_{U}^{\dagger}+6 \operatorname{tr}\left(m_{U} \Gamma_{U}\right)\left(m_{U} \Gamma_{U}\right)^{\dagger}$

$+6 i r \Gamma_{U}\left(m_{\nabla_{L}}^{2}+m_{U_{n}}^{2}\right) \Gamma_{U}^{\dagger}-2 g_{1}^{2}\left|m_{\lambda_{1}}\right|^{2}-6 g_{2}^{2}\left|m_{\lambda_{3}}\right|^{2}+g_{1}^{2} s$

Here 1 denotes the identity matrix with respect to the family indices.

\section{Appendix B. Approximate Analytic Solution}

We give the approximate analytical solution for the relevant parameters of the model. The approximations are justified in Sec. 3 and are of the following form:

$$
\begin{aligned}
\left(\Gamma_{E, U_{,} D}\right)_{14} & =h_{E, U, D} \gg\left(\Gamma_{E, U, D}\right)_{i i} ; \quad i=1,2,3 \\
g_{i} & =\frac{1}{3}\left[g_{i}\left(\mu_{R}=M_{W}\right)+g_{i}^{0}\right] ; \quad i=1,2,3 \\
m_{\lambda_{i}} & =\frac{1}{2}\left[\frac{g i\left(\mu_{\mathrm{K}}=M_{W}\right)^{2}}{7_{i}^{02}}+1\right] m_{\lambda}^{0} ; \quad i=1,2,3
\end{aligned}
$$

Here $(\Gamma E, U, D)_{i i}$ denote the Yukawe couplings for the $i^{\text {th }}$ family while $g_{1,2,3}$ and $m_{\lambda_{1}, \lambda_{2}, \lambda_{1}}$ are the gauyre couplings and the gaugino masses for $U(1)_{Y}, S U(2)_{L}$ and $S U(3)^{C}$ gauge groupa, respectively. The renormalization mass $\mu_{R}$ spans the range form the unification scale $M_{G}$ down to the weak scale $M_{W W}$. We use the following rotation

$$
\begin{aligned}
t & =\frac{1}{16 \pi^{2}} \ln \frac{\mu_{R}}{M_{G}} \\
X & =X_{0} \exp \left(14 \bar{g}^{2} t\right), \quad X_{0}=1-\frac{\bar{g}^{2}}{h_{0}^{2}} \\
h_{0} & =\frac{h_{U}^{0}+h_{D}^{0}}{2}, \quad Z_{0}=\frac{h_{U}^{0}-h_{D}^{0}}{h_{U}^{0}+h_{D}^{0}} \\
\bar{g}^{2} & =\frac{1}{7}\left(\frac{16}{3} g_{3}^{2}+3 g_{D}^{2} \quad \eta=\frac{g_{2}^{2}+g_{1}^{2}}{\bar{g}^{2}}\right. \\
J(x) & =\left\{1+\frac{4}{7} \frac{h_{E}^{02}}{\bar{g}^{2}} \int_{X}^{X_{0}} \frac{d X}{X}\left[\left(\frac{X}{X_{0}}\right)^{1-\eta}\left(\frac{1-X_{0}}{1-X}\right)\right]^{3 / 7}\right\}^{-1}
\end{aligned}
$$

Subscript 0 denotes the values of parameters at $\mu_{R}=M_{G}$. 
Yukatwa couplings of the fourth family $h_{E, U, D}$

$$
\begin{aligned}
& h_{U}^{2}=\frac{\bar{g}^{2}}{1-X}\left[1+2 Z_{0}\left[\frac{X}{X_{0}}\left(\frac{1-X_{0}}{1-X}\right)\right]^{5 / 7}\right] \\
& h_{D}^{2}=\frac{\bar{g}^{2}}{1-X}\left[1-2 Z_{0}\left[\frac{X}{X_{0}}\left(\frac{1-X_{0}}{1-X}\right)\right]^{5 / 7}\right] \\
& h_{E}^{2}=h_{E}^{02}\left[\left(\frac{X}{X_{0}}\right)^{1-\eta}\left(\frac{1-X_{0}}{1-X}\right)\right]^{3 / 7} J(x)
\end{aligned}
$$

Mass parameter $\mu$ of the superpotential

$$
\mu=\mu_{0}\left[\left(\frac{X}{X_{0}}\right)\left(\frac{1-X_{0}}{1-\bar{X}}\right)\right]^{3 / t} \exp \left(-3 g_{2}^{2} t\right)
$$

When evaluating the evolution of soft SS breaking parameters we neglected $h_{E}$ in comparison with $h_{U, D}$ and expanded in $\left(h_{U}-h_{D}\right) /\left(h_{U}+h_{D}\right)$.

Mass parameters $m_{H_{3}}, m_{H_{1}}^{2}$ and $m_{H_{2}}^{2}$

$$
\begin{aligned}
m_{H_{3}}= & m_{H_{s}}^{0}-\frac{6}{7}\left[m-\bar{m}_{\lambda}-\left(\bar{m}_{\lambda}-\frac{g_{2}^{2}}{2 \tilde{g}^{2}} m_{\lambda_{2}}\right) \ln \left(\frac{X}{X_{0}}\right)\right] \\
& +\frac{6}{7} \frac{X / X_{0}}{1-X}\left[\left(1-X_{0}\right)\left(m-\bar{m}_{\lambda}\right)+X_{0} \bar{m}_{\lambda} \ln \left(\frac{X}{X_{0}}\right)\right] \\
m_{H_{+}}^{2}= & \frac{m_{H_{1}}^{2}+m_{H_{3}}^{2}}{2}=-\frac{2}{7} m_{3 / 2}^{2}+\frac{6}{7} \tilde{m}^{2} \ell n\left(\frac{X}{X_{0}}\right)+\frac{3}{7} \Sigma \\
m_{H_{-}}^{2}= & \frac{m_{H_{3}}^{2}-m_{H_{2}}^{2}}{2}=\frac{10}{7} z_{0}\left[\frac{X}{X_{0}}\left(\frac{1-X_{0}}{1-X}\right)\right]^{3 / 7} \\
& \times\left\{\Sigma-\Sigma_{0}+\bar{m}^{2} \ln \left(\frac{X}{X_{0}}\right)+\frac{5}{7}\left[m_{+-}-m_{0}+\bar{m}_{\lambda} \ln \left(\frac{X}{X_{0}}\right)\right]^{2}\right\}
\end{aligned}
$$

where

$$
\begin{aligned}
\Sigma= & \left(\bar{m}_{\leq}^{2}-m_{\lambda}^{2}\right) \\
& +\frac{X / X_{0}}{1-\bar{X}}\left\{( 1 - X _ { 0 } ) \left[\Sigma_{0}-\left(m-\bar{m}_{\lambda}\right)^{2}\left(\frac{1-X / X_{0}}{1-X}\right)\right.\right. \\
& +2\left(m-\bar{m}_{\lambda}\right) \bar{m}_{\lambda} \frac{1}{1-X} \ln \left(\frac{X}{X_{0}}\right) \\
& \left.\left.-\left(\bar{m}^{2}-\bar{m}_{\lambda}^{2}\right)\right]+X_{0}\left[\bar{m}^{2}+\bar{m}_{\lambda}^{2} \frac{1}{1-X} \ln \left(\frac{X}{X_{0}}\right)\right] \ln \left(\frac{X}{X_{0}}\right)\right\}
\end{aligned}
$$

and

$$
\begin{aligned}
& \bar{m}_{\lambda}=\left(\frac{16}{3} g_{3}^{2} m_{\lambda_{3}}+3 g_{2}^{2} m_{\lambda_{2}}\right) /\left(\frac{16}{3} g_{3}^{2}+3 g_{2}^{2}\right) \\
& \bar{m}^{2}=\left[\frac{32}{3} g_{3}^{2} m_{\lambda_{3}}^{2}+6 g_{2}^{2} m_{\lambda_{2}}^{2}\right] /\left(\frac{16}{3} g_{3}^{2}+3 g_{2}^{2}\right) \\
& \widetilde{m}^{2}=\left[\frac{16}{3} g_{3}^{2} m_{\lambda_{3}}^{2}-\frac{1}{2} g_{2}^{2} m_{\lambda_{2}}^{2}\right] /\left(\frac{16}{3} g_{3}^{2}+3 g_{2}^{2}\right) .
\end{aligned}
$$

Here parameters $m_{Q_{L}, U_{R}, D_{R}}^{2}$ refer to the mass parameters corresponding to the fourth family. Subscript 0 denetes again the values of the corresponding parameters at $M_{G}$. Thus, $m_{H_{3}}^{0}, m_{0}$ and $m_{\lambda}^{0}$ are the parameters defined in Eq. (2.7) and $\Sigma_{0}=3 m_{3 / 2}^{2}$.

Soft supersymmetry breaking mass parameters $m_{U, D}$ and $m_{Q_{L}, U_{R}, D_{n}}^{2}$ corresponding to the fourth family

$$
\begin{aligned}
& m_{U} \approx m_{D}=\bar{m}_{\lambda}+\frac{X / X_{0}}{1-X}\left[\left(1-X_{0}\right)\left(m_{0}-\bar{m}_{\lambda}\right)+X_{0} \bar{m}_{\lambda} \ln \left(\frac{X}{X_{0}}\right)\right](B .8 a) \\
& m_{Q_{L}}^{2}=\frac{1}{7} m_{3 / 2}^{2}+\frac{2}{7} \Sigma-\frac{3}{7}\left(\tilde{m}^{2}+\frac{1}{2} \frac{g_{2}^{2}}{\bar{g}^{2}} m_{\lambda_{2}}^{2}\right) \ln \left(\frac{X}{X_{0}}\right) \\
& m_{U_{n}}^{2} \approx m_{D_{n}}^{2}=\frac{1}{7} m_{3 / 2}^{2}+\frac{2}{7} \Sigma-\frac{3}{7}\left(\tilde{m}^{2}-\frac{1}{2} \frac{g_{2}^{2}}{\bar{g}^{2}} m_{\lambda_{2}}^{2}\right) \ln \left(\frac{X}{X_{0}}\right)
\end{aligned}
$$

Mass parameters $\bar{m}_{\lambda}$ and $\tilde{m}^{2}$ are defined in Eqs. (B.7). 


\section{References}

1. E. Cremmer, S. Ferrara, L. Girardello, and A. Van Proeyen, Phys. Lett. 116B, 231 (1982): A. H. Chamseddine, R. Arnowitt, and P. Nath, Phys. Rev. Lett. 49, 970 (1982); R. Barbieri, S. Ferrara, and C. A. Savoy, Phys. Lett, 119B, 343 (1982); H. P. Nilles, M. Srednicki, and D. Wyler, Phys. Lett. 120B, 346 (1983) ; for a complete list of references see: H. P. Nilles, Phys. Rep. 110, 1 (1:334).

2. M. B. Green and J. H. Schwatz, Phys. Lett. 149B, 117 (1984) and CALT68-1224, December 1984.

3. J. H. Schwarz, Phys. Reports 89, 223 (1982); M. B. Green, Surveys in High Energy Physies 3, 127 (1983).

4. A. H. Chamseddine, R. Arnuwitt, and P. Nath, preprint NUB \# 2579 (1983); L. Hall, J. Lykken, and S. Weinberg, Phys. Rev. D27, 2359 (1983).

5. A. H. Chamseddine et al, Ref. 1; B. A. Ovrut and J. Wess, Phys. Lett. 112B, 347 (1982); L. E. Ibáiez, Phys. Lett. 118B, 73 (1982); for a complete list of referenees see: H. P. Nilles, Ref. 1.

6. J. M. Frère, D. Jones and S. Raby, Michigan preprint UMHE-82-5B.

7. L. Alvarez-Gaumé, J. Polchinski, and M. B. Wise, Nucl. Phys. B221, 499 (1983); J. Ellis, D. V. Nanopoulos, and K. Tamvakis, Phys. Lett. 121B, 123 (1983); L. E. Ibáñez and C. Lopéz, Phys. Lett. 13BB, 94 (1983) and CERN preprint TH^2650 (1983); L. Ibáñez, Madrid preprint FTUAM/83/4 (1983).

8. J. Ellis, J. S. Hagelin, D. V, Nanopoulos, and K. Tamvakis, Phys. Lett. 129B, 275 (1983); C. Kounsas, A. Lahanas, D. Nanopoulos, and M. Quiros, Phys. Lett. 132B, 95 (1983) and Nucl. Phys. B236, 438 (1984).

9. J. Ellis, A. B. Lahanas, D. V. Nanopoulos, and K. Tamvakis, Phys. Lett. 134B, 329 (1984); J. Ellis, C. Kounnas and D. V. Nanopoulos, CERN preprint TH-3768.

10. M. Cretič and J. C. Pati, Ihys. Lett. 135B, 57 (1984).

11. M. Cretic, SLAC-PUB-3568, February 1985, to be published in Phys. Lett $\mathbf{B}$

12. S. Coleman, and E. Weinberg, Phys. Rev. D7, 1888 (1973).

13. D. Gross, J. Harvey, E. Martinec and R. Rohm, Phys. Pev. Lett. 54, 502 (1985).

14. J. Bagzer, S. Dimopoulos and E. Massó, SLAC-PUB-3437, September 1984.

15. E. A. Paschos, Z. Phys. C26, 235 (1984).

16. J. W. Halley, E. A. Paschos, H. Usler, Phys. Lett. 155B, 107 (1984).

17. J. Bagger, S. Dimopoulos and E. Massó, SLAC-PUB-3693, May 1985; see also, J. Bagger, S. Dimopoulos and E. Masso, SLAC-PUB-3587, February 1985.

18. M. Goldhaber and W. Marciano, BNL-3587, April 1985.

19. J. P. Derendinger and C. A. Savoy, Nucl. Phys. B237, 30 t (1984).

20. See for example H. P. Nilles, Ref. 1.

21. S. P. Li and M. Sher, Phys. Lett. 140B, 339 (1984).

22. K. Inoue, A. Kakuto, H. Komatsu and S. Takeshita, Prog. Theor. Phys. 71, 413 (1984); see also Ref. 17 and L. Alvarez-Gaumé et al. in Ref. 9.

23. N. K. Falek, DO-TH 85/5, February 1985.

24. L. Hall, in Proceedings of the Winter School held in Mahabaheshwar, India (1984) 197. 


\section{FREE STRING FIELD THEORY}

$\therefore$

Why do we want to learn about string field theory? We have grown accustomed to thinking about physics at a fundamental level in terms of quantum field theory. So, whenever a new candidate for a replacement appears, one ought to check whether there is an appropriate correspondence, such that the usual picture of quanturn field theory emerges at least in the limit where we know that it describes nature. I believe string theory to be just such a candidate, and that its connection to quantum field theory should be made explicit. Of course, this has been done at the level of scattering amplitudes ${ }^{(1+)}$ long ago, and we know how to formulate string field theory on the light cone. ${ }^{[10]}$ However, this formalism does not lend itself easily to calculations, and our experience with light cone field theories leads us to believe that a covariant formulation will be clearer and simpler to calculate with. This should hold particularly for problems that require off-shell amplitudes, such as that of finding cosmological solutions or determining the structure of the Higgs sector. Also, since string theories encompass gravity, there should be a generalization of the equivalence principle, and on the light cone this is probably impossible to find. At the level of practicality, we would like to find a Feynman diagram technique for strings, that would render the integration over their modult spaces straightforward, if possibly tedioug.

The revival of string theories was accompanied by progress ${ }^{[16,20-30,2,4]}$ on the most pressing issues, the construction of gauge invariant interactions of open $^{[10,3 s, 0,0,7\}}$ bosonic and closed ${ }^{\mid 32, e .1\}}$ bosonic string fields and supersymmetric string field theory ${ }^{|22,0-18|}$ However, a complete and unified picture has not yet emerged and an efficient calculus is still missing.

As one step towards that goal we present the gauge invariant free string field theories, i.e. the open and closed bosonic, Neuveu-Schwarz (NS) and Ramond $(R)$ string field theortes. We fix them to the Feynman-Siegel and to the light cone gauge and obtain the known physical degrees of freedom, together with the appropriate Faddeey-Popov (FP) determinants ${ }^{|70|}$. Into the center of 
our formulation we put 2-dimensional BRST-invariance, with a natural calculus of differential forms ${ }^{[\omega \mid}$ axising from the creation and annihilation operatora of worldsheet ghosts. We obtain a simple formulation of free string field theory, in which the gauge invariant kinetic energy operator for open bosonic strings consists just of the 2-dimensional BRST charge $Q^{|31,1,-1 n|}$ We find that worldsheet and spacetime atatistics are related and that the GSO projection eliminates fields with the wrong spacetime statistics, such as physical vector particles behaving like fermions.

The chapter is organized as follows: First we introduce the calculus of forms mentioned sbove and decompose $Q$ into differential operators acting on forms. Then we present open bosonic strings in a formulation equivalent to that of ref. 23. , but simpler due to the introduction of additional auxiliary felds and the use of differential form language. We gauge fix explicitly to the Feynman-Siegel and to the light cone gauge in a fashion that easily generalizes to other string theories. We continue with a discussion of closed bosonic strings. In this case we modify the kinetic energy operator, changing the worldahcet BRST charge $Q$ into another operator $\tilde{Q}$ closely related to $Q$ that also satisfies $\tilde{Q}^{2}=0$. In addition, we require that our string fields be singlets under rotations on the worldsheet. This constraint may be derived ${ }^{1171}$ from an extended theory as an equation of motion, and we show how this is done. Now, in order to work out the NS sector of superstring field theory, one simply supersymmetrizes the differential forms obtained previously. In the $\mathbf{R}$ sector we encounter complications which originate in the zero mode structure of the string. As in the closed string case, we circumvent this problem by introduing \& kinetic operator $\tilde{Q}_{R}$ that satisfies $\tilde{Q}_{R}^{2}=0$ and is built out of pieces of $Q$. Yararon ${ }^{\text {(a) }}$ has proposed a gauge invariant action that properly includes all the zero modes, and we gauge fix this theory to the action based on $\tilde{Q}_{R}$. The last section will be devoted to closed superstrings. The construction of gauge invariant actions in the NS/NS zector is straightforward and for the NS/R ana $R / R$ sectors proceeds with the $\tilde{Q}$ - method. In the $R / R$ sector, this formulation requires an externally-imposed dynamical constraint. This is a shortcoming of our theory. Nevertheless, all of ous' string field actions are quantum-mechanically complete: in the light-cone gauge we recover the known physical spectra, without generating dynamical ghosts,

We adhere to the conventions of ref. 35 , as far as the mode decompsition and the (anti)commutators of quantum felds on the world sheet are concerned. 


\section{The String Exterior Derivative and the BRST Charge}

We define a string field to be a functional of the 2-dimensional quantum fields $x^{\mu}(z)$, the reparametrization ghosts $c(z)$ and $b(z)$ and, in the case of superstring theories, their superpartners $\psi^{\mu}(z), \gamma(z)$ and $\beta(z)$, with $z=\exp (r+i \sigma)$ for an Euclidean metric on the world sheet :

$$
\begin{aligned}
\mid \Psi) & \left.=\Psi\left[x^{\mu}, \psi^{\mu}, c, b, \beta, \gamma\right] \mid \Omega\right) \\
& \left.=\phi\left(x_{0}\right)+i \alpha_{-1}^{\mu} A_{\mu}\left(x_{0}\right)+c^{-1} \tau\left(x_{0}\right)+\cdots \mid \Omega\right)
\end{aligned}
$$

Here $\mid \boldsymbol{n})$ is the vacuum state satisfying $\phi_{n}|\Omega|=0$ for all annihilation operators $\phi_{n}$ in the theory and $x_{0}^{\mu}$ is the center of mass coordinate of the string. Of course, for bosonic strings there is no dependence on the world sheet fermion $\psi^{\mu}(z)$ and the ghosts $\gamma(z)$ and $\beta(z)$, and for closed strings we have to add in $\bar{x}^{\beta}(\bar{z}), \bar{\psi}^{\mu}(\bar{x})$ and so on. The mode expansion of $\psi, \gamma$ and $\beta$ is in integer powers of $z$ for the Neveu-Schwarz case, and in half-integer ones for Ramond boundary conditions; for example

$$
\begin{gathered}
\psi^{\mu}(z)=\sum_{n} z^{-n-\frac{1}{2}} \psi_{n}^{\mu} \quad ; \dot{n} \in \begin{cases}z+\frac{1}{2} & \text { (NS) } \\
z & (\mathrm{R})\end{cases} \\
\left\{\psi_{n}^{\mu}, \psi_{m}^{\mu}\right\}=\delta_{n+m, 0} \eta^{\mu \nu} \quad ; \eta^{\mu \nu}=\operatorname{diag}(-+++\cdots \dot{+}) .
\end{gathered}
$$

We distinguish the indices of $\psi, \gamma$ and $\beta$ from those of $x, c$ and $b$ by dotting them. The zero modes of the 2-dimensional fields deserve our special attention: we define $\mid \mathbf{\Omega})$ such that

$$
(n)=|\omega\rangle \otimes\left(\omega_{0}\right)
$$

where $(\omega)$ is the vacuum wavefunction for all the nonzero modes and, in the case of the Ramond superstring the zero modes of $\psi^{\mu}$, while the wavefunction for the ghost zero modes is denoted by $\left.\mid \omega_{g}\right)$. These states have the properties

$$
\begin{aligned}
(\omega \mid \omega) & =1 \\
\left(\omega_{g}\left|c^{0}\right| \omega_{g}\right) & =1
\end{aligned}
$$

and

$$
\left.b_{0}\left(\omega_{g}\right)=\beta_{0} \mid \omega_{g}\right)=0
$$

For comparison with ref. 35 , where the $S L(2)$-invariant vacuum $(0)$ is used, note that

$$
[\Omega]=c^{1}(0)
$$

for open bose strings,

$$
\left.\mid \mathbf{n})=c^{1} \mid q_{b c}=0, q_{\beta \gamma}=-1\right)
$$

for NS strings and

$$
\left.\mid \pi)=c^{1} \mid q_{b_{c}}=0, q_{\beta 7}=-\frac{1}{2}\right)
$$

in the $\mathrm{R}$ sector of superstrings.

For closed strings we drop all the zero modes, and instead we introduce a set of auxiliary modes $\widetilde{c}^{0}$ and $\tilde{b}_{0}$ which have the same anticommutators as $e^{0}$ and bo, and a vacuum state $(\Omega)$ with properties equivalent to (2.4) and (2.5) . A more detailed account of the zero modes in the Ramond sector is given below.

We find it useful to first expand $\Psi(\Omega)$ in ghost operators:

$$
\begin{aligned}
\Psi\{x, \psi, c, b, \gamma, \rho\}[\Omega]= & \left\{\Psi_{0}|x, \psi|+C^{\natural} \eta_{0}[x, \psi]+C^{-N} \Psi_{N}[x, \psi]+\ldots\right. \\
& \left.\left.+C^{-N_{b}} \cdots C^{-N_{2}} B_{-M_{-}} \cdots B_{-M_{1}} \Psi^{M_{1} \cdots M_{-}}{ }_{N_{1} \cdots N_{b}}\right] x, \psi\right] \\
& +\cdots\} \mid \Omega),
\end{aligned}
$$


where

$$
C^{-N} \in\left\{\mathrm{c}^{-n}, \gamma^{-\dot{n}}\right\}, B_{-N} \in\left\{b_{-n}, \beta_{-\dot{n}}\right\}, n \in \mathbf{Z}, \dot{\mathrm{n}} \in\left\{\begin{array}{ll}
\mathrm{Z}+\frac{1}{2} & \text { (NS) } \\
\mathrm{Z} & \text { (R) }
\end{array} .\right.
$$

The summations run over the indices appropriate for the string field under consideration.

We say that $\left.e^{-n_{1}} \cdots c^{-n_{3}} b_{-m_{4}} \cdots b_{-m_{3}}, W^{m_{1} \cdots m_{n_{1}} \cdots n_{1}}\right) \Omega$ is a $\left(\begin{array}{l}a \\ b\end{array}\right)$ form and, in the case of NS or $\mathbb{R}$ strings, $\left.C^{-N_{b}} \cdots B_{-M_{t}} W^{M_{1} \cdots M_{a}} N_{1} \cdots N_{b} \mid \Omega\right)$ is a $\left(\begin{array}{l}a \\ b\end{array}\right)$ superform. Forms are conveniently classified by their ghost number $\mathrm{g}$, which we define as follows:

$$
\begin{aligned}
& g(n)=0 \\
& g\left(c^{n}\right)=g\left(\gamma^{\frac{1}{n}}\right)=1 . \\
& g\left(b_{n}\right)=g\left(\beta_{n}\right)=-2 .
\end{aligned}
$$

The ghost number operator defined in this way is not hermitian; it satisfies $g^{t}=1-g$, but it acts on forms in a particularly simple way: a $\left(\begin{array}{l}a \\ b\end{array}\right)$ form has ghost number $g=b-a$. Note that a general form does not include any ghost zero mode. The zero modes play a special role and are therefore treated separately. String fields will in general contain zero modes, even if they have definite ghost number. For example, the fields that appear in the gauge invariant action are $\left(\begin{array}{l}k \\ k\end{array}\right)$ and $\left(\begin{array}{c}k+1 \\ k\end{array}\right)$ forms, for $k \in N_{0}$. They form a string field $\left.\left.\mid \Phi\right)=\left(\phi+c^{0} \eta\right) \mid \Omega\right)$ of ghost number 0 , which means $g(\phi)=0$ and $g(n)=-1$. We will sec that in the gauge fixed theory all possible forms appear.

Now, let us turn to the BRST charge on the world sheet. It is given by:

$$
\begin{aligned}
Q= & c^{-n} L_{n}+c^{0} l_{0}-\frac{1}{2} \gamma^{-\dot{n}} G_{\dot{n}} \\
& +\frac{1}{2} F_{m n}{ }^{k} c^{-n} c^{-m} b_{k}-\frac{1}{4} F_{\dot{m} n}^{k} \gamma^{-\dot{n}} \gamma^{-\dot{m}} b_{k}+\frac{3}{2} F_{m i n}{ }^{k} c^{-m} \gamma^{-\dot{n}} \beta_{k}
\end{aligned}
$$

where normal ordering is implied, the summations extend over $\mathbf{Z}$ or $\mathbf{Z}+\frac{1}{2}$ and
$F_{N M}{ }^{K}$ are the structure constants of the (super-) Virasoro algebr:

$$
\begin{array}{ll}
{\left[L_{n}, L_{m}\right]=F_{n m}{ }^{k} L_{k}+r_{n} \delta_{n+m, 0}=(n-m) L_{m+n}+r_{n} \delta_{n+m, 0}} \\
{\left[G_{n}, G_{m}\right]=F_{\dot{n} m}{ }^{k} L_{k}+r_{n} \delta_{n+m, 0}=2 L_{n+m}+r_{n} \delta_{\dot{n}+m, 0}} \\
{\left[L_{n}, G_{\dot{m}}\right]=F_{n \dot{m}}{ }^{k} G_{k}} & =\left(\frac{1}{2} n-\dot{m}\right) G_{n+m}
\end{array}
$$

With $r_{N}$ we denote the central charges:

$$
\begin{aligned}
& r_{n}= \begin{cases}\frac{D}{12} n\left(n^{2}-1\right) & \text { (bosonic strings) } \\
\frac{D}{8} n\left(n^{2}-1\right) & \text { (superstrings) }\end{cases} \\
& r_{i}=\frac{D}{2}\left(\dot{n}^{2}-\frac{1}{4}\right)
\end{aligned}
$$

and with la the Regge intercepts:

$$
l_{0}= \begin{cases}-1 & (\text { Bose }) \\ -1 / 2 & (\mathrm{NS}) \\ -D / 16 & (\mathrm{R})\end{cases}
$$

One can show that $Q^{2}=0$ precisely when the spacetime dimension is 26 for the bosonic strings and 10 for the superstrings.

If one separates the terms in $Q$ according to their zero mode content and their action on forms, one obtains

$$
Q=d+\delta+c^{0} K-2 b_{0} \Downarrow-\frac{1}{2} \gamma^{0} F+\frac{3}{2} \beta_{0} \downarrow-\frac{1}{4} \gamma^{0} \gamma^{0} b_{0}
$$

6 takes $\left(\begin{array}{l}a \\ b\end{array}\right)$ forms into $\left(\begin{array}{c}a-1 \\ b\end{array}\right)$ forms, whereas d maps $\left(\begin{array}{l}a \\ b\end{array}\right)$ forms into $\left(\begin{array}{c}a \\ b+1\end{array}\right)$ forms. $\Downarrow$ converts a $\left(\begin{array}{l}a \\ b\end{array}\right)$ form into a $\left(\begin{array}{c}a-1 \\ b+1\end{array}\right)$ form. It serves as an index lowering operator on our differential forms. $\downarrow$ is its supersymmetric complement. The differential operators $d$ and $\delta$ used here are very similar, but not exactly equal to those of 
ref. 23 . The difference lies in their normalization.

$$
K=L_{0}^{[x, \psi]}+N_{c}+N_{b}+N_{7}+N_{\mathcal{\beta}}
$$

is the Laplacian already encountered in ref. 23 . The operators $N_{b, e, \beta, 7}$ are the ghost mode equivalents of $L_{0}$. They count the sum of indices of $b, c, \beta$ and $\gamma$ respectively. Note that the terms in $Q$ containing $\gamma^{0}$ and $\beta_{0}$ are present only in the Ramond sector of the superstring. They contain the 2-d supersymmetric completion of the $c^{0}$ and $b_{0}$ terms in $Q$. Since worldsheet supersymmetry is explicitly broken by the NS boundary conditions on fermions, they do not appear in the BRST charge for the NS sector. The Dirac-Ramond operator

$$
F=G_{0}^{|x, \psi|}+\text { other terms }
$$

is the square root of $K$ in the same sense in which $\phi+m$ is the root of the Klein-Gordon operator $\square+m^{2}$, and satisfies $F^{2}=K$. Now, $Q^{2}=0$ implies

$$
\begin{aligned}
d^{2} & =\delta^{2}=0 \\
d \delta+\delta d & =2 K \downarrow+\frac{3}{4} F \downarrow \\
{[F, \downarrow] } & =-\frac{3}{4} \downarrow
\end{aligned}
$$

and all the other (anti-)commutators are zero. (2.19) implies

$$
d \delta+\delta d=F(F \Downarrow+\downarrow F)
$$

We will use this identity extensively in our treatment of the Ramond string. For the open bosonic string, (2.19) is just the algebsa laid out in ref. 23, apart from a different normalization. The scalar product between two string felds $\Phi$ and $\Psi$ will now bw written as

$$
(\Phi \mid \Psi)=\left(\Omega\left|\Phi^{t} \Psi\right| \Omega\right)
$$

With respect to this scalar product $d$ is the adjoint of $\delta$, while $K, F, \downarrow$ and $\downarrow$ are selfadjoint operators.

\section{The Open Bosonic String}

The formulation of Banks and Peskin ${ }^{[23\}}$ of the open bosonic string can be written in terms of the differential operators we just defined (note the change in the normalization of $d, \delta, K$ and $\Downarrow$ with respect to ref- 23). $A$ form of the open bosonic string theory considerably simpler than the original formulation of refs. 23-25 was independently discovered by Restuecia and Taylor, ${ }^{[14 \mid}$ Witten, ${ }^{[31]}$ Ramond, ${ }^{[0]}$ Neveu, Nicolaj and West, ${ }^{\mid 22]}$ and Aratyn and Zimerman. ${ }^{[10]}$

This simplified form of the theory has the virtue of bearing a much closer resemblance to Witten's interacting theory of open bose and fermi strings. ${ }^{[3,23)}$ The action is written as

$$
S=-\frac{1}{2}(\Phi|Q| \Phi)
$$

with

$$
\left.(\boldsymbol{\phi})=\boldsymbol{\Phi} \mid \mathbf{n})=\left(\phi+c^{D} \eta\right) \mid \mathbf{n}\right)
$$

a general string field of ghost number 0 . We obtain the following obvious gauge invariance:

$$
\begin{aligned}
& \delta_{\boldsymbol{x}}(\Phi)=Q(E), \\
& \mid E)=\left(t+t^{0} \theta\right)(\Omega), \\
& g(E)=-1,
\end{aligned}
$$

which has the following successive redundancies

$$
\begin{aligned}
& \left.\delta_{o}(E)=Q \mid G\right) \\
& \left.\delta_{H}(G)=Q \mid H\right\}
\end{aligned}
$$


Expanding (2.1) in ghost zero modes yields

$$
\begin{aligned}
S= & -\frac{1}{2}(\phi|K| \phi)-(\eta|\Downarrow| \eta) \\
& +\frac{1}{2}(\phi|(d+\delta)| \eta)+\frac{1}{2}(\eta|(d+\delta)| \phi),
\end{aligned}
$$

and the gauge transformations read

$$
\begin{aligned}
& \delta_{\infty \phi}=(d+\delta) \epsilon-2 \Downarrow \theta \\
& \delta_{\varepsilon \eta}=K \epsilon-(d+\delta) \theta .
\end{aligned}
$$

The $\epsilon$ transformation of $\phi$ displayed here is the gauge aymmetry identifed in refo. 23-25.

The action presented in refs. 23-25 may be obtained from (3.1) by gaugefixing some of the auxiliary fields which this action contains. In order to do that, we should recall from ref. 23 the concept of a maximally symmetrized form. Consider the coefficient $\phi^{M_{1} \ldots M_{4}} N_{1} \ldots N_{4}$ as a tensor with upper and lower indices, separately antisymmetrized. Imagine lowering the upper indices and then projecting the full set of indices onto combinations of definite symmetry. Because of the separate antisymmetrization, one may find only representations of the permutation symmetry corresponding to Young tableaux with two columns. The maximally symmetrized combination is defined to be the combination in which the second column is as long as possible, that is, in which as many lower indices as possible are symmetrized with upper indices, and vice versa. In a 0 form such as $\phi$, with equal numbers of upper and lower indices, the maximally symmetrized component is that in which every upper index is symmetrized with a lower index in the process of Young symmetrization. In general, maximally symmetrized forms with $g \geq 0$ are annihilated by $\Downarrow$, and form the kernel of this operator.
Let us, then, partially gauge-fix (3.5) by imposing $\forall(\phi)=0$. The resulting Fadeev-Popov determinant is nondynamical. Since $\Downarrow$ commutes with $d$ and $\delta$, we can see that $(d+\delta) \mid \phi)$ is a maximally symmetrized 1-form; thw, only the maximally symmetrized component of $|\eta\rangle$ couples to the remaining components of $\mid \phi)$. Since $|\eta\rangle$ is in any event nondynamical, we can freely drop (or integrate out) the other components, leaving only the maximally symmetrized one. This component is annihilated by $\Uparrow$, an operator encountered previously in ref.23. We define it as follows:

$$
\begin{gathered}
\Uparrow=\sum_{m \in N} \frac{1}{m} b_{-m} b_{m}-4 \sum_{A \in M} \beta_{-a} \beta_{a} \\
M= \begin{cases}N+\frac{1}{2} & \text { (NS) } \\
N & \text { (R) }\end{cases}
\end{gathered}
$$

Then, on string fields not containing zero modes, $[\Downarrow, \hat{\imath}]=n_{c}+n_{\gamma}-n_{b}-n_{\beta}=g$, where $n_{4}$ is the number operator for the modes of $\phi=b, c, \beta, \gamma$. In particular, for maximally symmetized forms of negative ghost number $\Uparrow$ is proportional to the left inverse of $\Downarrow:$ in our case $\uparrow \downarrow|\eta\rangle=\mid \eta)$. Using this relation to integrate out this last piece of $(\eta)$, we find at last

$$
S=-\frac{1}{2}(\phi|K| \phi)+\frac{1}{4}(\phi|(d+\delta) \Uparrow(d+\delta)| \phi) .
$$

which is the action of ref. 23 , written in our new conventions. Our gauge-fixing left the residual gange invariance:

$$
\left.\delta_{\varepsilon}(\phi)=(d+\delta) \mid \epsilon\right),
$$

where |E) has ghost number -1 and is restricted to be maximally symmetrized; this is precisely the gauge invariance of refs. 23-25. If we now use the results of ref. 26, we see that one can show the equivalence of $S$ in (2.1) to the open bosonic string in the light cone gauge ${ }^{\{10\}}$ and to Siegel's covariant gauge fixed action $^{[20]}$. 
In order to go to the Feynman-Siegel gauge, however, it is not necessary to take the above route. There is a shortcut, discovered by Witten ${ }^{(31 !}$ : the gauge fixing condition $\left.b_{0} \mid \Phi\right)=|\eta\rangle=0$ leads to the gauge fixed action

$$
\begin{aligned}
S_{Q f} & =-\frac{1}{2}(\Phi|Q| \Phi)+\frac{1}{2}\left(\bar{E}_{g}\left|b_{g} Q\right| E_{g}\right) \\
& =-\frac{1}{2}\left(\phi\left|c^{0} K\right| \phi\right)+\frac{1}{2}\left(\bar{\theta}_{g}\left|c^{0} K\right| \epsilon_{g}\right)-\frac{1}{2}\left(\bar{\theta}_{g}\left|(d+\delta) c^{0}\right| \theta_{g}\right)
\end{aligned}
$$

The ghost part possesses a gauge invariance of its own:

$$
\begin{aligned}
& \left.\delta_{a}\left(E_{q}\right)=Q \mid G\right) \\
& \delta_{\sigma}\left(\bar{E}_{\emptyset}\right)=0
\end{aligned}
$$

which we may $6 x$ by requiring $b_{0}\left|E_{q}\right|=\left|\theta_{0}\right|=0$. Then

$$
S_{\theta I}=-\frac{1}{2}(\Phi|Q| \Phi)+\frac{1}{2}\left(\bar{E}_{\theta}\left|b_{0}\right| E_{p}\right)-\frac{1}{2}\left(\bar{G}_{0}\left|b_{0}\right| G_{g}\right)
$$

The process continues and we finally end up with

$$
\left.S_{p f}=-\frac{1}{2}\left(\phi\left|c^{0} K\right| \phi\right)+\frac{1}{2}\left(\bar{\theta}_{\theta}\left|c^{0} K\right| \epsilon_{\theta}\right)-\frac{1}{2}\left(\bar{\kappa}_{q}\left|c^{0} K\right| \zeta_{q}\right)+\cdots\right]
$$

where $g(\phi)=0, g\left(\bar{\theta}_{q}\right)=1, g\left(\epsilon_{g}\right)=-1, g\left(\bar{\kappa}_{g}\right)=2, g\left(\zeta_{q}\right)=-2, \ldots$. This, of course, constituter exactly the field content and the form of the action found by Siegel $^{\mid \text {|al| }}$. The BRST invariances are

$$
\begin{aligned}
& \left.\left.\delta_{\text {DNST }} \mid \phi\right)=(d+\delta) \mid \epsilon_{g}\right) \\
& \delta_{\text {DSST }}\left(\epsilon_{g}\right)=0 \\
& \delta_{\text {DAST }}\left(\bar{\theta}_{p}\right)=0,
\end{aligned}
$$

replacing the gauge invariance of $\mid \Phi)$,

$$
\begin{aligned}
& \left.\left.\delta_{\text {aRsT }} \mid \epsilon_{g}\right)=(d+\delta) \mid \zeta_{g}\right) \\
& \left.\delta_{\text {DRST }} \mid \zeta_{g}\right)=0 \\
& \left.\left.\delta_{\text {DRST }} \mid \kappa_{g}\right)=-(d-\delta) \mid \bar{\theta}_{g}\right) \\
& \left.\delta_{\text {aRST }} \mid \bar{\theta}_{g}\right)=0
\end{aligned}
$$

replacing the gauge invariance of $\left.\mid E_{G}\right)$, and so on. The $\epsilon$ transformation of $\dot{\phi}$ displayed here is the gauge symmetry identified in refs, 23-25.

Let us now discuss the gauge-fixing of (3.1) to the light-cone gauge. The action of the open string in the light-cone gauge is given by

$$
S_{\ell \sigma}=-\frac{1}{2}\left(\phi_{t}|K| \phi_{t}\right) \text {, }
$$

where $\phi_{t}$ contains only transverse states. To characterize these states, let us denote the light-cone components of $\alpha_{N}^{\mu}$ by

$$
K_{N}=\alpha_{N}^{+}, \quad M_{N}=\alpha_{N}^{-}
$$

With this rotation, the transverse states are those which include no $K, M, B$, or $C$ creation operitors acting on $(0)$. We must, then, show that all states other than the transverse states may be removed from (3.1) by a choice of gauge. To do this, we will use a counting argument similar in form to the one developed in sef. 26 to discuss the gauge fixing of the action of refs, 23-25. (The reader who finds this argument a bit sketchy should consult ref. 26 for a more discursive presentation.)

Represent the classes of states we must gauge away as:

$$
\left.K^{P} C^{q} M^{r} B^{s} \mid 0\right)
$$

where $p, q, r, s$ denote the number of creation operators of the given type which act on $(0), p+q+\gamma+s=N>0$. Since at any given mass level, $\mathcal{N}$ has a maximum 
value, we can confine our attention to states with a fixed value of $N$, beginning at the maximum, and sequentially remove all of these states from (3.1). We can remove these fields without generating Fadeev-Popov determinants if we shift by terms in (3.6) which involve no factors of $p^{-}$. We will, in fact, use only terms in (3.6) involving $(d+\delta)$. We will only need to consider the term in $d$ of the form

$$
d=C^{-N} \cdot p^{+} M_{N}+\ldots
$$

and the term in $\delta$ of the form

$$
\delta=C^{N} \cdot p^{+} M_{-N}+\ldots
$$

we may imagine, then, that $d$ simply converts a $K$ to a $C$ and $\delta$ simply converts a $B$ to an $M$.

As a simple illustration of the use of these rules, let us discuss the counting of gauge parameters for states with $N=1$ and 2 . For states with $N=1$, the only gauge parameters are of the form $B \mid 0$ ). These suffice to gauge away all states in $\mid \phi)$ of the form $M(0)$. The remaining states in $|\phi\rangle$ which we need to eliminate are those of the form $K \mid 0)$. These states appear together with the states $M \mid 0$ ) in the first term of (3.5), but this term has been removed by our choice of gauge. The only remaining place that the states $K(0)$ appear is in the cross terms of (3.5); since $d$ converts a $K$ to a $C$, this state can overlap with states $B \mid 0$ ) in $\mid \eta$ ). This matrix element uses only the term (3.19) in $d$, which contains no $p^{-}$. Thus, the states $K \mid 0)$ act as Lagrange multipliers to eliminate the states $B(0)$ in $\mid \eta)$. Thus, we have exactly the gauge freedom we require to eliminate all states with $N=1$.

The analogous argument for $\mathcal{N}=2$ illustrates some complications found at higher levels. The states in $(\phi)$ and $\mid \eta)$ which must be eliminated have the form

$$
\left.\left.\left.\left.\left.K^{2} \mid 0\right), K M \mid 0\right), M^{2} \mid 0\right), \quad K B \mid 0\right), M B|0\rangle, \quad B C \mid 0\right)
$$

The gauge parameters in $(\epsilon)$ and $(\theta)$ have the form

$$
\left.K B \mid 0), M B \mid 0), \quad B^{2} \mid 0\right)
$$

In addition, we must consider the gauge parameters of the gange parameters, which characterize the redundancies in (3.22). These are states in $\mid G)$, of the form

$$
\left.B^{2} \mid 0\right)
$$

It is useful to think of these multiplets of states as components of tensors whose indices run over all positive integers. The commutation relations of these operators place restrictions on these tensors: $\left.B^{2} \mid 0\right)$ is antisymmetric in its indices, and $\left.M^{2} \mid 0\right)$ is symmetric. Thus, we can use (3.23) to gauge away the antisymmetric part of $M B \mid 0)$ in (3.22); the remaining symmetric part of this multiplet can gauge away the states $M^{2} \mid 0$ ) in (3.21). $\left.K B \mid 0\right)$ in (3.22) can gauge away $K M \mid 0$ ), and $\left.B^{2} \mid 0\right)$ in (3.22) can gauge away the antisymmetric part of $\left.M B \mid 0\right)$ in (3.21). The remaining states in (3.21) are either Lagrange multipliers or are eliminated by Lagrange multipliers: $K^{2} \mid 0$ ) eliminates the symmetric part of $M B \mid 0$ ), and $K B \mid 0)$ eliminates $B C \mid 0)$.

Let us now generalize this counting argument to all levels. As a first step, we must reduce the full set of gauge parameters in $|E\rangle$ to those parameters which cannot be gauged away by higher-level gauge transformations. Consider, for exsmple, the components of $\mid E$ ) of the form

$$
\left.K^{p} C^{q} M^{r-1} B^{s+1} \mid 0\right)
$$

Some of these components can be removed by acting with $\delta$ on components of $\mid G$ ) of the form $\left.K^{p} C^{q} M^{r-2} B^{s+2} \mid 0\right)$. These components have their own redundancies, corresponding to the states $K^{P} C^{q} M^{r-3} B^{s+3} \mid 0$ ), and so forth. The nonredundant components of $\mid E$ ) can be identified as follows: Operators $M^{*}$ form an $r$-index 
symmetric tensor with indices in the sat of values of $N(N>0)$. Similarly, operetors $B^{\prime}$ form an s-index antisymmetric tensor. It is convenient to project states with both $M$ 's and $B$ 's onto states of definite (mixcd) permutation symmetry, labeled by Young tablcawx. For example, $\left.M^{4} B^{3} \mid 0\right)$ belongs to

$$
\square+B=F^{\square}+F^{D} \text {. }
$$

Since we will be sceing many products of this form, let us refer to a Young tableau of $r$ symmetrized boxes as $\{r\}$, a tableau of $s$ antisymmetrized boxes as $[s]$, and a tableau with a row of $r$ boxes above a column of $s$ boxes as $(r / s)$. In this language, (3.25) reads

$$
\{4\} \times[3]=(5 / 2)+(4 / 3) \text {. }
$$

One can then see that states (3.24) in $\mid E)$ contain $M$ 's and $B$ 's in the representation $(r / s)+(r-1 / s+1)$. Their redundancies belong to $(r-1 / s+1)+(r-2 / s+2)$. The redundancies of the redundancies belong to $(r-2 / s+2)+(r-3 / s+3)$. Continuing until one runs out of $M^{\prime}$ 'B, and then resolving the net effect of these parameters, one finds that the nonredundant component of the gauge parameters in (3.24) have $M$ 's and $B$ 's combined to the symmetry $(r / s)$.

We will act on $\mid \Phi)$ with these symmetry motions in a different way depending on whetises or not $r \geq p$. If $r \geq p$, act $\delta$ on the nonredundant components of (3.24) to remove states of the form (3.18). The piece of (3.18) which remains has $M$ 's and $B^{\prime} \mathrm{s}$ symmetrized according to $(r+1 / s-1)$, so that the full set of operators displayed has the character

$$
\{p\} \times|g| \times\{r+1 / s-1\} \text {. }
$$

If $r<p$, decompose $\{p\} \times[q] \rightarrow(p+1 / q-1)+(p / q)$. Act $\delta$ on the $(p+1 / q-1)$ component to remove states of the form $\left.K^{p} C^{q} M^{\prime} B^{0} \mid 0\right)$. Act $d$ on the $(p / q)$ component, to remove states of the form $\left.K^{p-1} C^{q+1} M^{r-1} B^{8+1} \mid 0\right)$. The effect of this transformation is to reduce each group of states $\left.K^{P} C^{q} M^{r} B^{*} \mid 0\right)$ with $r<p$ to the structure:

$$
(p / q) \times(r+1 / s-1)+(p / q) \times(r / s)=(p / q) \times\{r\} \times[s] .
$$

Now let us examine the form of (3.1) that we have obtained. We have gauged away all states with $q=s=0, r \geq p$. Thus, the states with $q=s=0$, $\tau<p$ cannot appear in the first, diagonal term of (3.5). They can only appear in the off-diagonal terms involving $(d+\delta)$, using a $d$ to convert it to the structure $K^{p-1} C^{1} M^{r} \mid 0$ ), which has a nonzero matrix element with states if the form $\left.K^{r} M^{p-1} B^{1} \mid 0\right)$. As in our simple examples above, the terms with $q=s=0$ act as Lagrange multipliers which elimainate terms with $s=1$. After the gauge transformations described in the previous paragraph, both sets of states have been reduced to the multiplet $(p / 0) \times\{r\}$, so all of the remaining states of the form (3.18) with $q=0, s=1$, and $r \geq p$ are eliminated. Now the states with $q=0, s=1, r<p$ appear only as Lagrange multipliers for the states with $q=1, s=1, r \geq p$. Comparing the representations into which these have been projected, we see that all of these states are eliminated. The pattern continues until all components of $(\Phi)$ have either been removed or have acted as Lagrange multipliers to remove others.

In comparing this argument to that of ref. 26 , the reader should note that here we find no nondynamical component fields in addition to the transverse felds. All unwanted components of $|\Phi\rangle$ disappear. It is never necessary to use the fact that the $\mid \eta)$ components are purely auxiliary. This last feature is essential for generalizing this argument to the theories we will consider in Section 6 . 


\section{The Closed Bosonic String}

It is possible to construct a gauge invariant theory of closed strings along the lines of the previous section. However, just enlarging the index space to include barred operators and replacing $Q$ in (3.1) by the BRST operator for the closed string does not work. The physical spectrum has to obey the algebraic constraint $K-\bar{K}^{*}=0$, and this condition is not a consequence of the Ansatz. The complications arise from the zero mode structure of the ghosts. We may circumvent these dificulties by just dropping all the zero mode dependence of the string fields and introducing instead two formal operators $\tilde{b}_{0}$ and $\tilde{c}^{0}$ with the same algebra as $b_{0}$ and $c^{0}$ :

$$
\left(\tilde{b}_{0}\right)^{2}=\left(\tilde{c}^{0}\right)^{2}=0,\left\{\tilde{b}_{0}, \tilde{c}^{0}\right\}=1 .
$$

Then, let us define the vacuum to have a structure analogous to the open string case, eq. (2.4). We build all our string fields on this vacuum, and impose the constraint

$$
(K-\bar{K})(\Phi)=0 .
$$

Henceforth we will work in the subspace of string fields obeying this condition. Now replace $Q$ by

$$
\tilde{Q}=c^{0}(K+\bar{K})+d+\delta+\bar{d}+\bar{\delta}-2 b_{0}(\Downarrow+\bar{W})
$$

Then it is easily verified that $\widetilde{Q}^{2}=0$ and therefore

$$
S_{c}=-\frac{1}{2}(\Phi|\tilde{Q}| \Phi)
$$

with $\left.\mid \Phi)=(\phi)+\tilde{\tau}^{0} \mid \eta\right)$ is gauge invariant just. like in the open string case. Note that the constraint $K-\bar{K}$ commutes with all the operators we encountered in our discussion of the open string as well as with their barred counterparts. Therefore, the gauge fixing procedures to the Feynman-Siegel gauge and to the light cone gauge can now be taken verbatim from the section on open strings. Again we find Siegel's gauge fixed action ${ }^{120}$, but without the Lagrange multipliess that enforce $K=\bar{K}$. This constraint, even though it is algebraic, is one of the constraints ore encounters when first-quantizing the closed string, and hence it should be regarded as an equation of motion for second-quantized string felds. Therefore we would like to find a formulation of closed string field theory that leads to this equation of motion. This has been done by Ballestrero and Maina ${ }^{[171}$ and we will describe their construction in the following.

For that purpose, let us change our notation from barreu and unbarred operators $a$ and $\bar{a}$ to the linear combinations $a^{ \pm}=1 / \sqrt{2}(a \pm \bar{a})$. Also, let $D=d+\delta+\bar{d}+\bar{b}$. Then the BRST charge is

$$
Q_{c}=Q+\bar{Q}=c^{0+} K^{+}+c^{0-} K^{-}+D-2 b_{0}^{+} \Downarrow^{+}-2 b_{0}^{2} \Downarrow^{-} .
$$

We keep the complete zero mode structure and build out of a linear combination of the ghost zero mode states $\left.\left.\left.\mid \omega_{g}\right) \otimes\left(\bar{\omega}_{0}\right), c^{0}\left(\omega_{0}\right) \otimes \mid \bar{\omega}_{0}\right), \vec{c}^{0} \mid \omega_{0}\right) \otimes\left(\bar{\omega}_{0}\right)$, and $c^{0} \bar{c}^{0}\left(\omega_{\sigma}\right) \otimes\left(\bar{\omega}_{g}\right)$ a vacuum state $\left(\Omega_{0}\right)$ that satisfies

$$
c^{0-}\left(\Omega_{0}\right)=0, \quad b_{0}^{+}\left(\mathbf{\Omega}_{g}\right)=0
$$

and

$$
\left(\Omega_{g}\left|c^{0+} b_{0}^{-}\right| \Omega_{g}\right)=1 .
$$

Explicitly, $\left(\cap_{g}\right\}=c^{0-}\left(\omega_{g}\right) \otimes\left(\bar{\omega}_{g}\right)$, and we build string fields on the vacuum state $|\Omega(t)=| \omega|\otimes| \Omega_{0} \mid$. Then the action

$$
S=\frac{1}{4 \sqrt{2}}\left[\left(\Phi|| b b_{0}^{-}, Q_{c}|| \Phi\right)+\left(\Phi\left|Q_{c} K^{-}\right| \Sigma\right)-\left(\Sigma\left|K^{-} Q_{c}\right| \Phi\right)\right]
$$

is gauge invariant under

$$
\begin{aligned}
& \left.\delta_{\boldsymbol{L}}|\Phi\rangle=Q_{e} \mid \Phi\right) \\
& \delta_{\Lambda}(\Sigma)=(\Phi) .
\end{aligned}
$$


Now, let us show the equivalence of (4.8) to (4.4). We use the components of (A) that do not satisfy $K^{-}=0$ to fix the partial gauge $\left.K^{-} \mid \Sigma\right)=0$. This leads a nondynamical Fadeev-Popov determinant, which we may normalize to 1 . In other words, we do not generate ghosts by this procedure. The $(\Sigma)$-dependent terms in the action vanish and if we now expand $\mid \Phi)$ in the ghost zero modes,

$$
\left.\mid \Phi)=\left(\phi+\sqrt{2} c^{0+} \eta+\frac{1}{\sqrt{2}} b_{0}^{-} x+c^{0+} b_{0}^{-} \psi\right) \mid \Omega\right),
$$

we obtain

$$
\begin{aligned}
S=\frac{1}{2 \sqrt{2}}[ & -\left(\phi\left|K^{+}\right| \phi\right)+\sqrt{2}(\eta|D| \phi)+\sqrt{2}(\phi|D| \eta)-4\left(\eta\left|\Downarrow^{+}\right| \eta\right) \\
& \left.+\frac{1}{2}\left(\phi\left|K^{-}\right| \psi\right)+\frac{1}{2}\left(\psi\left|K^{-}\right| \phi\right)+\frac{1}{2}\left(\eta\left|K^{-}\right| x\right)+\frac{1}{2}\left(x\left|K^{-}\right| \eta\right)\right] .
\end{aligned}
$$

This action has exactly the form we wanted to get: the fields $|\psi\rangle$ ) and $|\chi|$ are Lagrange multipliers enfoscing $K^{-}=0$, and if we integrate them out, we obtain precisely (4.4) in component form, as one easily recognizes by comparing (4.11) with (4.4) and (3.5).

\section{Neveu-Schwarz Strings}

One abtains the Neuveu-Schwarz sector of the superstring by a straightforward supersymmetrization of the bosonic string. In the construction of superforms (2.9) we now sum over dotted and undotted indices, Our forms then differ from the ones found in supersymmetry textbooks ${ }^{\{30 \mid}$ only by the rule $\left[c^{n}, \gamma^{\text {in }}\right]=0$, whereas the standard choice reads $\left\{d x^{n}, d \theta_{\dot{n}}\right\}=0$.

A general field $\mid \Phi)$ now contains as expanison coefficients tensor fields of different statistics. In

$$
\begin{aligned}
\mid \Phi)= & \left\{x(x)+\psi_{-\frac{1}{2}}^{\mu} A_{\mu}(x)-i \beta_{-\frac{1}{2}} \bar{c}(x)-\gamma^{-\frac{1}{2}} c(x)\right. \\
& \left.\left.-\alpha_{-1}^{\mu} V_{\mu}(x)+\frac{1}{2} \psi_{-\frac{1}{2}}^{\mu} \psi_{-\frac{1}{2}}^{\mu} T_{\mu \nu}(x)+\ldots\right\} \mid \Omega\right)
\end{aligned}
$$

let us choose $A_{\mu}(x)$ to be bosonic, since we wish to describe spacetime bosons in the NS sector. Then $\mid \Phi$ ) must be a Grassmann-valued form. For the ghost field $c(x)$ and the antighost $\bar{c}(x)$ this works out nicely, since they have fermi statistics. However, the fields $\chi(x), V_{\mu}(x)$, and $T_{\mu \nu}(x)$ are also assigned Grassmann values, even though the corresponding forms have ghost number $D$, and the fields are therefors physical. They have integer spin and then clearly the wrong statistics. We will have to project out these unwanted fields. In (\$), they arise as expansion coefficients of products of creation operators with an even number of creation operators $\xi^{n} \in\left\{\psi_{\mu}^{\dot{n}}, \gamma^{\dot{n}}, \rho_{\dot{n}}\right\}, \dot{n} \in N-\frac{1}{2}$. Obviously, the projection to use is just the good old GSO one $e^{|s|}$,

$$
P_{\text {aso }}=(-)^{n_{\gamma}+n_{7}+n_{A+1}}=1 \text {. }
$$

The remarkable correlation between two-dimensional and space-time statistics first appeared in Siegel's papers ${ }^{\text {(20) }}$ on the gauge-fixed bosonic string theory. The observation that the GSO projection must be made in order to preserve the correct statistics of fields in the Neveu-Schwarz-Ramond theory has also been made 
by LeClair. ${ }^{\text {(') }}$ For properly GSO-projected string fields it is now straightforward to supersymmetrize the preceding two sections. We simply use instead of the operators $Q, \tilde{Q}$ and $Q$, their natural extensions to the NS sector. The ghost zero mode structure is the same as before, and therefore all the manipulations go through as deacribed above.

The Neveu-Schwarz-Ramond theory contains three types of closed strings, those with Neveu-Schwarz boundary conditions for both left- and right-movers, those with Ramond boundary conditions for one set of modes, and those with Ramond boundary conditions for both left- and right-moving modes. The sectors of the first and third type lead to bosonic string states; however, it is convenient to treat the third type together with the fermionic strings. We are ready, though, to write the action for the first sector. In fact, this action is exactly (4.4), with the bosonic string operstors $d, \delta, K, \Downarrow$ replaced by their Neveu-Schwarz counterparts and with a GSO projection applied independently to the leit- and right-moving components of each form. This projection does not affect the proof of gauge invariance or the process of gauge-fixing, both of which proceed exactly as above. By replacing only the left-moving operators by Neveu-Schwarz operators, while keeping the right-moving operators those of the bosonic string, we find a free field action for the bosonic states of the heterotic string. ${ }^{(3) \mid}$

\section{Ramond Strings}

In the Ramond sector, things get a little mure complicated due to the existence of zero modes in the fermion sector on the worldsheet. In particular, in addition to $c^{0}$ and $b_{0}$ there now exist ghost zero modes $\gamma^{0}, \beta_{0}$ with bose statistics. Also, we have to cope with the zero mades of $\psi^{\mu}\{x\}$. We do this by gplitting 'the 10 fermionic operators $\psi_{0}^{\psi}$ into 5 creation and 5 annihilation operaturs. The ground state is then 32-fold degenerate, and for Euclidean spacetime we denote it as the spinor $\mid \alpha) \in 16+\overline{16}$ of $S O(10)$. An even number of $/ \mathrm{g}$ 's generates the 16 , i.e. lefthanded spacetime spinors, while applying $\psi_{0}$ 's an odd number of times yields the $\overline{16}$, which describes righthanded particles. The vacuum with respect to the $\psi_{0}$ 's is then the highest weight state of the 16 of SO(10), and the $\psi^{\mu}$ zero modes act on it as $\Gamma$-matrices. If we represent the operators $\psi_{0}^{\mu}$ in matrix form, we will implicitly multiply all operators with worldsheet formi statistics with $(-)^{n_{0}}=\Gamma^{11}$ in order to maintain the proper anticommutation relations. The hermiticity properties of the BRST-charge and its components follow from $\Gamma^{\mu f}=\Gamma^{0} \Gamma^{\prime 2} \Gamma^{0}$ and are given by ,

$$
Q^{\dagger}=\Gamma^{0} Q \Gamma^{0}, F^{\dagger}=\Gamma^{0} F T^{0}, d^{\dagger}=\Gamma^{0} \delta \Gamma^{0}, \ldots
$$

Note that for $\mathrm{SO}(10)$ the spinor representations are complex, while in Minkowski spacetime, i.e. for $S O(1,9)$, these representations say be chosen to be real Majorana spinors.

As in the Neveu-Schwarzsector the GSO projection ensures the proper spacetime statistics of the component fields. For example, in coder to define a general string field

$$
\left|\Psi^{a}\right\rangle=\left\{\xi^{a}+c^{a} \eta^{a}+\ldots|\Omega\rangle\right.
$$

with uniform statistics, we have to project $\xi^{a}$ such that it has definite spacetime helicity. The choice we make then requires $\eta^{a}$ to have the opposite helicity. 
Therefore the GSO projection must fix the sign of

$$
P_{\text {oso }}=(-)^{n_{b}+n_{c}+n_{4}} \text {, }
$$

where the number operators include the zero modes. When we choose to : vark in the $\Gamma$-representation of the $\psi^{\mu}$ zero modes, we simply replace their contribution to $P_{\text {oso }}$ by $\Gamma^{11}$.

In order to write down an action for $R$ strings, we employ the same trick we used for closed bose strings. We drop all the ghost zero modes and replace them by $\tilde{c}^{0}$ and $\tilde{b}_{0}$. We modify $P_{o s o}$ accordingly and are again able to contruct a nilpotent operator

$$
\tilde{Q}_{n}=\tilde{c}^{0}(-)^{f} F+d+\delta-\tilde{b}_{0}(-)^{\mathcal{T}}(F \Downarrow+\Downarrow F) .
$$

The operator $(-)^{f}$ is inserted to make $\tilde{Q}$ anticommuting. By definition it anticommutes with any 2-d feld with fermi statstics, and hence it may be chosen to be identical to $P_{\text {ogo }}$. Our Ansatz for the Ramond action is then

$$
S=-\frac{i}{\sqrt{2}}\left(\bar{\Phi}\left|\tilde{Q}_{R}\right| \Phi\right)
$$

where $\left.\mid \Phi^{a}\right)=\left(\phi^{a}+\hat{\partial}^{0} \eta^{a}\right)|\Omega|$ is a $g=0$ string field that $s i$ : hes $P_{G s o}=1$ and $(\bar{\Phi}|=| \Phi)^{\dagger} \mathbf{r}^{0}$.

This action has the gauge invariance $\left.\left.\delta_{w} \mid \Phi\right)=\dot{\psi}_{x}, n_{j}\right)$, whe $\mathrm{I}$ is now a spinor-valued $g=-1$ field. By directly applying the steps $; \quad$ is; $\quad$ om $(3,10)$ to (3.13) , this action can be gauge-fixed to the Feynman-Siegel gauge

$$
S_{p s}=-\frac{i}{\sqrt{2}}(\bar{\phi}|\stackrel{\dot{F}}{\mid}| \phi),
$$

where now $(\phi)$ is a general $P_{\text {aso }}=+1$ form in the space of nonzero modes. Using the arguments given for the descent to the light-cone gauge, (6.6) can also be gauge-fixed to the form

$$
S_{L 0}=-\frac{i}{\sqrt{2}}\left(\bar{\phi}_{t}\left|G_{0}\right| \phi_{t}\right)
$$

where $\left.\mid \phi_{t}\right)$ is a transverse state. From this formula, one can then easily reach the light-cone gauge action by integrating out the components of $\left|\phi_{t}\right\rangle$ satisfying $\left.\Gamma^{+} \mid \phi_{t}\right)=0$.

Written in components (after performing the zero mode algebra), the action (6.7) takes the form

$$
\begin{aligned}
S=-\frac{i}{\sqrt{2}}\{(\phi|F| \phi)+(\bar{\eta}|F \Downarrow+\Downarrow F| \eta) \\
-(\bar{\phi}|d+\delta| \eta)-(\bar{\eta}|d+\delta| \phi)\} .
\end{aligned}
$$

Note that in this expression, unlike the bosonic string actions, the auxiliary field (7) has become dynamical. This turns out to have no efict on the gauge-fixing of the action by the methods of Section 3 ; our arguments there did not make use of the explicit form of the term quadratic in the auxiliary feld. However, it is interesting to note that this fect does play a role in more conventional, component-by-component covariant gauge-fixing. As a concrete example, let us consider the first excited mass level. The components of $|\phi\rangle$ at this level are the vector-spinor coeficients of the states $\alpha_{-1}^{\mu}|0\rangle$ and $\left.\psi_{-1}^{\mu} \mid 0\right)$; these have opposite chirality and thus can form a massive spin- $\frac{3}{2}$ field. In fact, after redefining these component fields appropriately, one recovers the action for a gravitino made massive by compactifying a spin- $\frac{3}{2}$ field in 11 dimensions on a circle. The conventional covariant quantization of this field would bring in 3 massive spin- $\frac{1}{2}$ ghosts, the third being the Nielsen-Kallosh ghost. ${ }^{|3 c, 30|}$ The Feynman-Siegel gauge action for the Ramond string contains only two massive ghosts. But (6.8) also contains, at this level, two dynamical components of $|n\rangle$, corresponding to the atates $b_{-1}|0\rangle$ and $\beta_{-1}|0\rangle ;$ these have opposite chirality and combine to form a massive spin- $\frac{1}{2}$ 
fermion with normal statistics. This fermion precisely compensates the Nielsen-

\section{Kallogh ghost.}

We have now reached an understanding of how the physical Ramond spectrum may be generated by a covariant field theory. What we are still lacking is a clear pieture of the ghost 2ero modes. We expect that the reduction of the zero mode space to $\tilde{c}^{0}$ and $\tilde{b}_{0}$ bears some resemblance to the case of closed bose strings. However, in the Ramond sector this problem is substantially more difficult: not oniy is the Hilbert space infinite dimensional, being generated by the commuting operator $\gamma^{0}$, but in addition there exist an infinite number of inequivalent copies. These copies are called 'bose seas' and are characerized by integers $M$. All $\gamma^{\text {th }}$ with $\dot{n} \leq M$ act on the $M$-vacuum as creation operators, and the remaining $\gamma^{\dot{n}}$

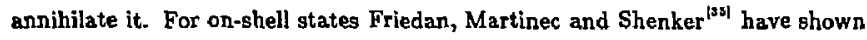
the equivalence of all the bose seas, in the sense that scattering amplitudes for physical states may be evaluated in any one of them, and the result is independent of the bose sea chosen for the computation. This proof does not hold for off-shell states, and we are left with the so-called bose sea problem: how do the bose seas enter the field theory and what does the reduction to (6.8) look like?

A partial answer to this question has been girven by Yamron ${ }^{\mid 30 !}$, who constructed a free field theory in one bose sea. We will describe his formulation briefly and gauge fix his action to (6.8). First, let us discuss some properties of the bose ghost zero modes. They satisfy $\left[\gamma^{0}, \beta_{0}\right]=1$, and we will work in the base sea where $M=0$. Its vacuum $\left.\mid \omega_{b}\right)$ is annihilated by $\beta_{0}$ and therefore the zero mode Hilbert space $H$ is the space of functions of $\gamma^{0}$. The vacuum state is represented as the constant function 1 , and $\beta_{0}$ acts like a derivative: $\beta_{0}=-\boldsymbol{\gamma}_{7^{0}}$. The inner product of $H$ must be defined in such a way that $\beta_{0}$ is antihermitian: $\left\langle\omega_{b}\right|=\int d \gamma^{0}$. This implies

$$
\left(\omega_{b}\left|\delta\left(\gamma_{0}\right)\right| \omega_{b}\right)=1
$$

The Ansatz for the action is

$$
S=-i \sqrt{2}(\bar{\Phi}|G Q| \Phi)
$$

where $Q$ is the BRST charge (2.16) and $G$ is an operator that acts like a metric. In order to obtain a nonzero inner product, $G$ must contain the function $\delta\left(\gamma^{0}\right)$, and since we want a first order action, a factor $c^{0}$ should appear. The gauge invariance

$$
\left.\delta_{\infty}(\Phi)=Q \mid E\right)
$$

is obvious if $\{G, Q\}=0$. Yamron has constructed a metric satisfying all these requirements. In our convention it reads

$$
G=c^{0} \sum_{n=0}^{\infty} \frac{1}{(2 n+1) !}(-8 \downarrow)^{n} \partial_{\gamma^{0}}^{2 n+1} \delta\left(\gamma^{0}\right)=2 e^{0} \delta\left(\left(\gamma^{0}\right)^{2}+8 \Downarrow\right) .
$$

A comparison of (6.12) with (2.16) shows that the terms in $Q$ that contain $b_{0}$ do not appear in $S$. Consequently, the string field cornponents which contain $e^{0}$ are projected away by $G$. Then the expansion of $(\Phi)$ in ghost zero modes has the form

$$
\left.[\Phi)=\left(\phi+\frac{1}{2} \gamma^{0} \eta+\left(\gamma^{0}\right)^{2} A_{D}+\left(\gamma^{0}\right)^{3} A_{1}+\left(\gamma^{0}\right)^{1} A_{2}+\ldots\right) \mid \Omega\right),
$$

and the gauge parameter $(E)$ may be written as

$$
\left.|E\rangle=\sum_{m=0}^{\infty} E_{m}\left(\gamma^{0}\right)^{m}[\Omega)+\sum_{n=0}^{\infty} D_{n} c^{0}\left(\gamma^{0}\right)^{n} \mid \Omega\right)
$$

We now use the gauge parameters $D_{n}, n \geq 0$ to set $A_{n}=0, n \geq 0$. The corresponding gauge transformations are

$$
\left.\left.\delta \mid A_{n}\right)=-\frac{1}{4} \mid D_{n}\right)-2 \Downarrow\left(D_{n+2}\right),
$$

and we see that the corresponding Fadeev-Popov determinant is algebraic, so that no dynamical ghosts are generated. If one now performs the $\gamma^{0}$-algebra in 
(6.10), then $S$ reduces exactly to (6.8), and we have arrived at our original formulation of open Ramond strings.

The fermionic closed string theories can be constructed along the same basic lines. The elosed strings with Ramond left-movers and Neveu-Schwarz rightmovers can be written for string forms satisfying the constraint (4.2). Define

$$
\widetilde{Q}=\tilde{c}^{0}(-)^{f} F+d+\delta+\bar{d}+\bar{\delta}-\tilde{b}_{\mathrm{b}}(-)^{f}(F \Downarrow+\Downarrow F+F \bar{\Downarrow}+\bar{\Downarrow} F) .
$$

Then the appropriate action is given by $(6.7)$, where now $\mid \Phi)$ is a string form built on the product space of left- and right- movers, GSO projected independently in each subspace. The fermionic heterotic string action is constructed in the same way, using the bosonic string operators to build the right-moving subspace.

Finally, we turn to the closed superstring theory corresponding to Ramond boundary conditions for both left- and right- movers. In this sector, our simplistic treatment of the zero modes breaks down. We have been able to construct a quantum-mecharically complete theory, but this theory has two defects. First, it requires a constraint which, in a general frame, is dynamical. Second, it re quires that part of the GSO projection be done after quantization rather than before. Despite these defects, we are encouraged to present this formulation because does generalize the formal structure we have set out for the other strings, and because it continues out formulation of the other closed superstrings in a suggestive pattern.

The basic fields in this sector will be string fields carrying two Dirac indices and satisfying the condition

$$
(F-\bar{F})(\beta)=0 \text {. }
$$

Since $F^{2}=K$, this condition implies (4.2). However, while (4.2) is a purely algebraic condition, this condition contains time derivatives of $(\beta)$. Choose

$$
\tilde{Q}=\tilde{c}^{0}+(d+\delta+\bar{d}+\bar{\delta})-\tilde{b}_{0}(F(F \Downarrow+\Downarrow F)+\vec{F}(\overline{F \Downarrow}+\overline{\Downarrow F})),(6.18)
$$

where $\tilde{b}_{0}, \tilde{c}^{0}$ satisfy (4.1) and have odd GSO parity with respect to both the left-moving and the right-moving GSO operators $P_{a s o}$ and $\bar{P}_{\text {aso. }}$. Define

$$
\left.\left.\left.\mid B)=(\mid \boldsymbol{\beta})+\vec{c}^{0} \mid \rho\right)\right) \mid \boldsymbol{\Omega}\right)
$$

and

$$
(\overline{\bar{B}})=\left(B \mid \Gamma^{0} \overline{\Gamma^{0}}\right.
$$

Then the gauge-invariant action for this sector may be written

$$
S=\frac{1}{4}(\overline{\bar{B}}|\tilde{Q}| B)
$$

(B) should be restricted to have the correct statistics: $P_{n s o} \cdot \bar{P}_{a s a}=1$. However, if we apply at this point the separate conditions $P_{a s o}=\bar{P}_{a s o}=1$, the chirality conditions do not match and (6.20) vanishes. Note that the closed superstring charges that we have defined (eqs. (4.3), (6.16), (6.18)) fall into a simple pattern.

Despite the fact that the constraint (6.17) is dynamical in a general frame, we can quantize this system straightforwardly by observing that, in the light-cone frame, (6.17) becomes a set of nondynamical relations. To make this point clear, we will discuss in a very explicit way the quantization of the massless level of this string. This level contains antisymmetric tensor fields, and so one would suspect that it should have a gauge invariance. In our formulation, however, there is no gauge invariance; the required reduction of degrees of freedom is implemented by the dynamical constraint. (The constraint (5.10) looks suggestively like a gaugefixing condition for a Duffin-Kemmer Lagrangian.) The light-cone quantization of the remaining levels will then fo'low by analogous manipulations, after fixing of the light-cone gauge for the oscillators in the mwner of Section 3. 
Choose the following representation of the $\Gamma^{\mu}$ matrices:

$$
\Gamma^{+}=\left(\begin{array}{cc}
0 & -i \sqrt{2} \\
0 & 0
\end{array}\right), \Gamma^{-}=\left(\begin{array}{cc}
0 & 0 \\
i \sqrt{2} & 0
\end{array}\right), \Gamma^{i}=\left(\begin{array}{cc}
i \gamma_{8}^{i} & 0 \\
0 & -i \gamma_{8}^{i}
\end{array}\right)
$$

where $y_{b}^{i}$ are a set of real symmetric Dirac matrics of $O(8)$. Express the massless level of $(\beta)$ is $\mathrm{b}=\beta \mathrm{r}^{0} ; \mathrm{b}$ trassforms under Lorentz trassformations like a Dirac matrix. The action (6.21), restricted to this level, takes the form

$$
S=\frac{1}{4} \operatorname{tr}\left[\Gamma^{0} \mathbf{b}^{T} \Gamma^{0} \mathbf{b}\right]
$$

Decompose $b$ in the basis of eq. (6.22), as follows:

$$
\mathbf{b}=\left(\begin{array}{ll}
\mathbf{b}_{\uparrow} & \mathbf{b}_{+} \\
\mathbf{b}_{-} & \mathbf{b}_{\downarrow}
\end{array}\right) \text {. }
$$

On the massless level, $\mathbf{F}=-\sqrt{2} \partial$, so (6.17) may be written:

$$
p b=-b \mathbf{p} .
$$

Let $\hat{p}=p^{i} \gamma_{i}^{i}$. Then the full content of $(6.25)$ is expressed by the relations:

$$
\begin{aligned}
& \sqrt{2} p^{+} \mathbf{b}_{\downarrow}=-\sqrt{2} p^{+} \mathbf{b}_{\uparrow}-\left[\hat{p}, \mathbf{b}_{-}\right] \\
& \sqrt{2} p^{+} \mathbf{b}_{+}=\sqrt{2} p^{-} \mathbf{b}_{-}-\left\{\hat{p}, \mathbf{b}_{\uparrow}\right\} .
\end{aligned}
$$

Use these equations to eliminate $b_{1}$ and $b_{+}$in $(6.23)$. Then $b_{\uparrow}$ may be seen to be auxiliary and can be integrated out. This reduces $(6.23)$ to the form

$$
S=\frac{1}{2} \operatorname{tr}\left[\mathbf{b}_{-T}^{T} \frac{p^{2}}{2 p^{+}} \mathbf{b}_{-}\right] \text {. }
$$

If one now imposes the chirality conditions on $\mathbf{b}$. which follow from $\mathbf{G}=\overline{\mathbf{G}}=\mathbf{1}$, we are let with a theory of a doubly chiral $O(8)$ bispinor. This is the correct physical content for the massiess section of the Ramond/Ramond closed string.
To generalize this discussion to higher mass levels of the string, we need two observations. First, the light-cone gauge-fixing procedure of Section 3 still allowg us to remove all states with longitudinal, timelike, and ghost excitations. Then the quantization procedure reduces to the treatment of the explicit Dirac indices. On higher levels, (6.17) equates two massive Dirac operators. The mass terms always couple two different field components which have opposite chirality but the same GSO parity. Thus, each massive Dirac operator may be written as the action on a pair of Dirac spinors of the operator

$$
\left(\dot{\boldsymbol{\rho}} \boldsymbol{\rho}+M \mathrm{~T}_{M}\right)
$$

where $\Gamma_{M}$ anticommutes with the $\Gamma^{\mu}$. (If these massive equations follow by dimensional reduction, in the manner suggested by Siegel and Zwiebach, ${ }^{1 \cdot{ }^{\prime \prime}}{ }_{2}^{\prime} M=$ $\Gamma^{11}$.) Then the analysis of the previous paragraph can be repeated for every massless level by treating the mass term in (6.28) as an extra component of the transverse momentum. This demonstration completes our formulation of free field theories, which can be explicitly gauge-fixed to the known physical apectra, for all of the known stringe and superstrings. 


\section{References}

1. S. Raby, R. Slansky, G. West, Los Alamos preprint LA-UR-85-3794 (1985).

- 2. J.-L. Gervais, Ecole Normale Superieure preprint LPTENS 85 (1985).

3. D. Pfeffer, R. Ramond, V. Rogers, University of Florida preprint UFTP85-19.

4. P. Ramond, University of Florida preprint UFTP-85-18 (1985).

5. H. Hata, K. Itoh, T. Kugo, H. Kunitomo, K. Ogawa, Phys. Lett. 172B , 186 (1986) , Phys. Lett. 172B , 196 (1986) .

6. A. Neveu, P.C. West, Phys. Lett. 188B , 192 (1986) ,

7. M. A. Awada, Phys. Lett. 172B , 32 (1986) .

8. A. LeClair, Phys. Lett, 168B , 53 (1986) , A. LeClair, J. Distler, Nucl. Phys. B27s , 552 (1986).

9. H. Ooguri, Phys. Lett. 172B, 204 (1986) .

10. Y. Kazama,A. Neveu, H. Nicolai, P. C. West, CERN preprint CERN-TH4301/85 (1985)

11. S.P. de Alwis, N. Ohta, Phys. Lett. 174B , 383 (1986) ,Phys. Lett. 174B , 388 (1986) .

12. G.D. Date, M. Günaydin, M. Pernici, K. Pilch, P. van Nieuwenhuizen, Physs. Lett. I71B , 182 (1986) .

13. E. Witten, Princeton University preprint Print-86-0250.

14. H. Aratyn, A. H. Zlmerman, Nucl. Phys. B269, 349 (1986), Ghosts and the Physical Modes in the Covariant Free String Field Theory, Hebrew University preprint, RIP-85-9 (1985) , Phys. Lett. 168B , 75 (1986) , Phys. Lett. 160B, 130 (1986) .

15. S. Uehara, H. Terao, Phys. Lett. 168B , 70 (1986) , Phys. Lett. 173B , 134 (1986) , Phys. Lett. 173B , 409 (1986).
16. A. Restuccia, J. G. Taylor, King's College preprint (1985) .

17. A. Ballestrero, E. Maina, Turin University preprint DFTT-16/86 (1986).

18. T. Yoneya, Prog. Theor. Phys. 51 , 1907 (1974) , J. Scherk, J. Schwarz, Nuel. Phys. B81, $118(1974)$.

19. See chapters 10 and 11 in J. Schwarz, Superstrings: the first 15 years of superstring theory, World Scientific, 1985

20. W. Siegel, Phys. Lett. 151B , 391 (1985) , Phys. Lett. 151B , (396) 1985 .

21. D. Friedan, Phys. Lett. 162B , (102) 1985 ; String Field Theory, Chicago University preprint EFI 85-27, 1985

22. A. Neveu, P.C. West, Phys. Lett. 165B ,63 (1985) , A. Neveu, J. Schwarz, P.C. West, Phys. Lett. 164B , 51 (1985) , A. Neveu, P.C. West, Nucl. Phys. B268, 125 (1986) , A. Neveu, H. Nicolai, P.C. West, Nucl. Phys. B204, 573 (1986).

23. T. Banks, M. Peskin, Nuel. Phys. B264, 513 (1986).

24. W. Siegel, B. Zwiebach, Nucl. Phys. B263, 105 (1986) , B. Zwiebach, MIT preprint MIT-CPT-1308(1985) .

25. K. Itoh, T. Kugo, H. Kunitomo, H. Ooguri, Prog. Theor. Phys. T5 , 162 (1986) .

26. M. Peskin, C. Thorn, Nucl. Phys. B269, 509 (1986) .

27. K. Bardakei, Nucl. Phys. B271, 561 (1986) .

28. C. Thorn, Phys. Lett. 159B, 107 (1985) , Addendum, Phys. Lett. 160B , 430 (1985) .

29. M. Kaku, Introduction to the Field Theory of Strings, New York City College preprint CCNY-HEP-85-11 (1985); Supergauge Field Theory of Covariant Heterotic Strings, Osaka University preprint OU-HET-79 (1985); 
Phys. Lett. 162B, 97 (1985); Nucl. Phys. B276 , 125 (1986) ; M. Kaku and J. Lykken, Supergauge Field Theory of Superstrings, New York City College preprint Print-85-412 (1985) .

30. A. Cohen, G. Moore, P. Nelson, J. Polchinski, An Invariant String Propaqator, University of Texas preprints UTTG-26-85 (1985); Nucl. Phys. B287, 143 (1986) ; J. Polchinski, Comm. Math. Phys. 104, 37 (1986) .

31. E. Witten, Nucl. Phys. B268, 253 (1986) .

32. A. A. Tseytlin, Phys. Lett. 168B , 63 (1986) .

33. F. Gliozzi, J. Scherk, D. Olive, Nucl. Phys. B122, 253 (1977) .

34. J.P. Yamron, Phys. Lett. 174B , 69 (1986) .

35. D. Friedan, E. Martinec and S. Shenker, Nucl. Phys. B271, 93 (1986) .

36. J. Wess, J. Bagger, Supersymmetry and Supergravity, Princeton University Press, 1983; S.J. Gates, M.T. Grisaru; M. Roxek, W. Siegel, Superspace, Benjamin-Cummings, 1983 .

37. D.J. Gross, J.A. Harvey, E. Martinec and R. Rohm, Hetenotic String Theory. 2. The Interacting Heterotic String, Princeton University preprint Print-85-0694 (1985), Nucl. Phys. B256, 253 (1985).

38. N. K. Nielsen, Nucl. Phys. B140, 477 (1978).

39. R. Kallosh, Nucl. Phys. B141, 141 (1978).

40. L. Baulieu, Physics Reports 129,1 (1985), and references therein . 